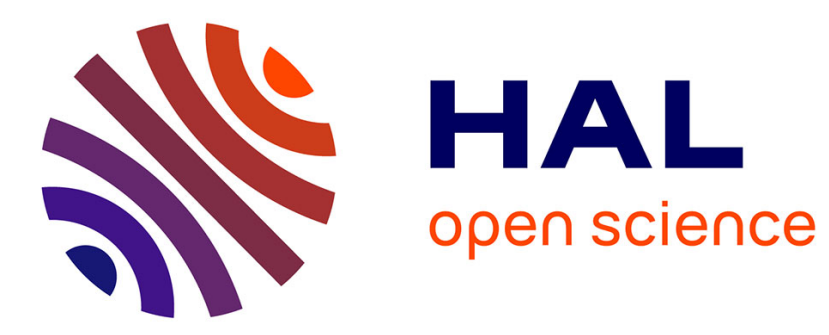

\title{
On the Relationship between the TKE Dissipation Rate and the Temperature Structure Function Parameter in the Convective Boundary Layer
}

Hubert Luce, Lakshmi Kantha, Hiroyuki Hashiguchi, Abhiram Doddi, Dale Lawrence, Masanori Yabuki

\section{To cite this version:}

Hubert Luce, Lakshmi Kantha, Hiroyuki Hashiguchi, Abhiram Doddi, Dale Lawrence, et al.. On the Relationship between the TKE Dissipation Rate and the Temperature Structure Function Parameter in the Convective Boundary Layer. Journal of the Atmospheric Sciences, 2020, 77 (7), pp.2311-2326. 10.1175/JAS-D-19-0274.1 . hal-03211339

\section{HAL Id: hal-03211339 \\ https://hal.science/hal-03211339}

Submitted on 3 Dec 2021

HAL is a multi-disciplinary open access archive for the deposit and dissemination of scientific research documents, whether they are published or not. The documents may come from teaching and research institutions in France or abroad, or from public or private research centers.
L'archive ouverte pluridisciplinaire HAL, est destinée au dépôt et à la diffusion de documents scientifiques de niveau recherche, publiés ou non, émanant des établissements d'enseignement et de recherche français ou étrangers, des laboratoires publics ou privés. 


\title{
On the Relationship between the TKE Dissipation Rate and the Temperature Structure Function Parameter in the Convective Boundary Layer
}

\author{
HUBert LuCE \\ Université de Toulon, Aix-Marseille University, CNRS IRD, MIO, UM110, La Garde, France \\ LAKSHMI KANTHA \\ Department of Aerospace Engineering Sciences, University of Colorado Boulder, Boulder, Colorado \\ HIROYUKI HASHIGUCHI \\ Research Institute for Sustainable Humanosphere, Kyoto University, Kyoto, Japan \\ ABHIRAm DodDi AND DALE LAWRENCE \\ Department of Aerospace Engineering Sciences, University of Colorado Boulder, Boulder, Colorado \\ MASANORI YABUKI \\ Research Institute for Sustainable Humanosphere, Kyoto University, Kyoto, Japan
}

(Manuscript received 7 October 2019, in final form 13 April 2020)

\begin{abstract}
Under stably stratified conditions, the dissipation rate $\varepsilon$ of turbulence kinetic energy (TKE) is related to the structure function parameter for temperature $C_{T}^{2}$, through the buoyancy frequency and the so-called mixing efficiency. A similar relationship does not exist for convective turbulence. In this paper, we propose an analytical expression relating $\varepsilon$ and $C_{T}^{2}$ in the convective boundary layer (CBL), by taking into account the effects of nonlocal heat transport under convective conditions using the Deardorff countergradient model. Measurements using unmanned aerial vehicles (UAVs) equipped with high-frequency response sensors to measure velocity and temperature fluctuations obtained during the two field campaigns conducted at Shigaraki MU observatory in June 2016 and 2017 are used to test this relationship between $\varepsilon$ and $C_{T}^{2}$ in the CBL. The selection of CBL cases for analysis was aided by auxiliary measurements from additional sensors (mainly radars), and these are described. Comparison with earlier results in the literature suggests that the proposed relationship works, if the countergradient term $\gamma_{D}$ in the Deardorff model, which is proportional to the ratio of the variances of potential temperature $\theta$ and vertical velocity $w$, is evaluated from in situ (airplane and UAV) observational data, but fails if evaluated from large-eddy simulation (LES) results. This appears to be caused by the tendency of the variance of $\theta$ in the upper part of the CBL and at the bottom of the entrainment zone to be underestimated by LES relative to in situ measurements from UAVs and aircraft. We discuss this anomaly and explore reasons for it.
\end{abstract}

\section{Introduction}

For locally homogeneous, stationary and isotropic turbulence produced by shear flow instabilities in a stably stratified atmosphere, turbulence kinetic energy (TKE) dissipation rate $\varepsilon$ and the structure function parameter for temperature $C_{T}^{2}$ are theoretically interrelated.

Corresponding author: Hubert Luce, luce@univ-tln.fr
The expression is (e.g., Tatarskii 1961, 73-76; Gossard et al. 1998; Kantha and Luce 2018)

$$
\varepsilon=\left(\alpha \frac{g}{T^{2}} \frac{C_{T}^{2}}{N^{2}}\right)^{3 / 2}
$$

where $g$ is the gravitational acceleration, $T$ is the temperature, $N^{2}=(g / \theta)(d \theta / d z)$ is the squared Brunt-Vaïsälä (buoyancy) frequency, $\theta$ is the potential temperature 
and the parameter $\alpha=\left(1 / \beta_{\theta}\right)\left[\left(1-R_{f}\right) / R_{f}\right],{ }^{1}$ where $\beta_{\theta} \approx$ 3.0 is a universal constant and $R_{f}$ is the flux Richardson number given by the ratio between buoyancy flux and turbulent energy production.

Kantha and Luce (2018) and Luce et al. (2019) tested this theoretical relationship in the free troposphere and in clear air conditions, from direct estimates of $\varepsilon$ and $C_{T}^{2}$ from high-resolution wind and temperature measurements made by unmanned aerial vehicles (UAVs) during the Shigaraki UAV-Radar Experiment (ShUREX) 2016 and 2017 campaigns [see Kantha et al. (2017) for detailed description of the campaigns]. The study confirmed the statistical validity of Eq. (1), with $\alpha \approx 0.17$ (corresponding to $R_{f} \sim 0.14$ ) consistent with similar observations in the ocean (Kantha and Luce 2018).

Because the energy and flux budgets are different in the CBL due to different sources of instabilities and turbulence (mainly buoyancy-driven from the bottom due to solar heating), we expect Eq. (1) does not apply to $\mathrm{CBL}$, at least for the well-mixed portion of the CBL, where $N^{2}$ is expected to be nearly zero and in the lower part of the CBL, where it is negative. Luce et al. (2019) reported that the largest statistical disagreement between the direct and indirect estimates of $\varepsilon$ using Eq. (1) were indeed observed inside the CBL. In addition to theoretical problems, the evaluation of Eq. (1) on individual profiles is made difficult by the fact that measuring local values of $N^{2}$ is not easy in presence of thermal updrafts and downdrafts (see appendix of Luce et al. 2019).

The behavior of $C_{T}^{2}$ in CBL has been extensively studied in the past by theoretical and experimental approaches (e.g., Kaimal et al. 1976; Fairall 1987; and references therein). However, to the authors' knowledge, the relationship between $\varepsilon$ and $C_{T}^{2}$ in the CBL has not been addressed. In the present work, we propose an analog to Eq. (1) for convectively driven turbulence, by assuming the validity of Deardorff's $(1966,1972)$ approach for correcting for nonlocal effects on the vertical heat flux in the central, well-mixed part of CBL. The correction consists of adding a countergradient term $\gamma$ to the sensible heat flux equation based on $K$ theory. We tentatively express $\gamma$ in terms of $\varepsilon$ and $C_{T}^{2}$. These two parameters can be accurately estimated from highresolution measurements of winds and temperature (e.g., Luce et al. 2019), so that the proposed theoretical

\footnotetext{
${ }^{1}$ We point out that $\alpha$ is generally noted by $\gamma$ in the literature (e.g., Kantha and Luce 2018; Luce et al. 2019). The notation has been changed in order to avoid confusion with the countergradient term expressed in (5).
}

relationship between these two parameters can be experimentally verified.

The derivations of the desired expression, based on the heat flux budget equation and on the parameterizations made by Deardorff (1972) for the well-mixed part of the CBL, are described in section 2. Section 3 describes briefly the instrumentation used for collecting observational data and presents the methods and criteria used for selecting CBL cases used for the present study. Section 4 describes the results of experimental evaluation of the expression proposed in section 2, from comparisons between UAV data and data disseminated in the literature, mainly airplane observations and largeeddy simulations (LES). These comparisons highlighted significant differences between temperature fluctuation intensity parameters, $C_{T}^{2}$ and $\left\langle\theta^{2}\right\rangle$, estimated from in situ measurements and those predicted by LES in the upper part of the well-mixed region and in the entrainment zone of the CBL. These differences suggest that the effects of entrainment on $C_{T}^{2}$ and $\left\langle\theta^{2}\right\rangle$ are more pronounced in the atmosphere than as depicted in LES results. Although inconsistencies between in situ observations and LES made comparison and interpretation difficult, we make the assumption that the in situ measurements are more representative of the CBL dynamics and structure than the LES model results. If this is true, then the Deardorff model of countergradient term would be quantitatively relevant, in spite of the deficiencies in its formulation. These issues are discussed in section 5 .

\section{Theory}

\section{a. Sensible heat flux equation and countergradient terms}

From the sensible heat flux equation, assuming horizontally homogeneous conditions, no subsidence, and a steady state under Boussinesq approximation, we have (e.g., Stull 1988)

$$
\frac{\partial\langle w \theta\rangle}{\partial t}=0=\underbrace{-\left\langle w^{2}\right\rangle \frac{\partial \Theta}{\partial z}}_{M} \underbrace{-\frac{\partial\left\langle w^{2} \theta\right\rangle}{\partial z}}_{T} \underbrace{-\frac{1}{\rho_{0}}\left\langle\theta \frac{\partial p}{\partial z}\right\rangle}_{P} \underbrace{+\frac{g}{\theta_{0}}\left\langle\theta^{2}\right\rangle}_{B} .
$$

The angle brackets $\langle\cdots\rangle$ indicate spatial averages. The four terms on the right-hand side are as follows: $M$ is the so-called mean gradient production term, $T$ is the vertical turbulent transport term, $P$ is the pressure gradient-potential temperature covariance term, and $B$ is the buoyancy production term. The variables are as follows: $\Theta(z)$ is the background (mean) potential 
temperature, $p$ is pressure perturbation, $\theta_{0}$ is the mean potential temperature in the $\mathrm{CBL}$, and $\left\langle\theta^{2}\right\rangle(z)$ and $\left\langle w^{2}\right\rangle(z)$ are the variance of turbulent potential temperature and vertical wind fluctuations, respectively. Terms $T$ and $P$ are third moments and must be parameterized by suitable closure schemes.

By neglecting $T$ from arguments based on observations and by using the Rotta "return to isotropy" closure scheme for $P$,

$$
P=-\frac{\langle w \theta\rangle}{\tau},
$$

where $\tau$ is a pressure relaxation time scale, Deardorff $(1966,1972)$ showed that Eq. (2) can be written as

$$
\langle w \theta\rangle=-K_{H}\left(\frac{\partial \Theta}{\partial z}-\gamma_{\mathrm{D}}\right),
$$

where $K_{H}=\left\langle w^{2}\right\rangle \tau$ is the turbulent diffusivity and $\gamma_{\mathrm{D}}$ is a positive term called "countergradient term" given by

$$
\gamma_{\mathrm{D}}=\frac{g}{\theta_{0}} \frac{\left\langle\theta^{2}\right\rangle}{\left\langle w^{2}\right\rangle} .
$$

Equation (4) indicates that the sensible heat flux (based on $\theta$ ) in the well-mixed part of the CBL is not only due to the usual local downgradient transport but also to a nonlocal convective transport $\gamma_{\mathrm{D}}$. The nonlocal term $\gamma_{\mathrm{D}}$ expresses the vertical redistribution of the surface flux of $\theta$ upward by convective eddies, independently of the local gradient of $\theta$. The term $\partial \Theta / \partial z-\gamma_{\mathrm{D}}$ can be seen as an apparent (negative) potential temperature gradient, which justifies the countergradient heat transport in the upper half of the CBL, where the temperature gradient is expected to be stable (e.g., Deardorff 1966; Stull 1988). However, it is worth noting that the Deardorff formulation ignores the turbulent transport term $T$ in the vertical heat flux budget, even as it is trying to account for heat transport by large eddies in the CBL.

Deardorff's approach was widely accepted, until numerical simulations of CBL dynamics from LES made evaluations of each term of Eq. (2) possible. Figure 1 shows vertical profiles of $M, T, P$, and $B$ according to LES performed by Holtslag and Moeng (1991, hereafter referred as HM91) and Ghannam et al. (2017, hereafter referred as GH17) for strongly convective cases, in which buoyancy production of TKE dominates shear production.

The profiles shown in Fig. 1 are normalized by scaling variables used by similarity theory for strong convection. First, $z_{i}$ is the CBL depth defined as the altitude of the minimum of (negative) heat flux, or, in practice as the height of the bottom of the capping temperature inversion. The depth of the well-mixed potion of the

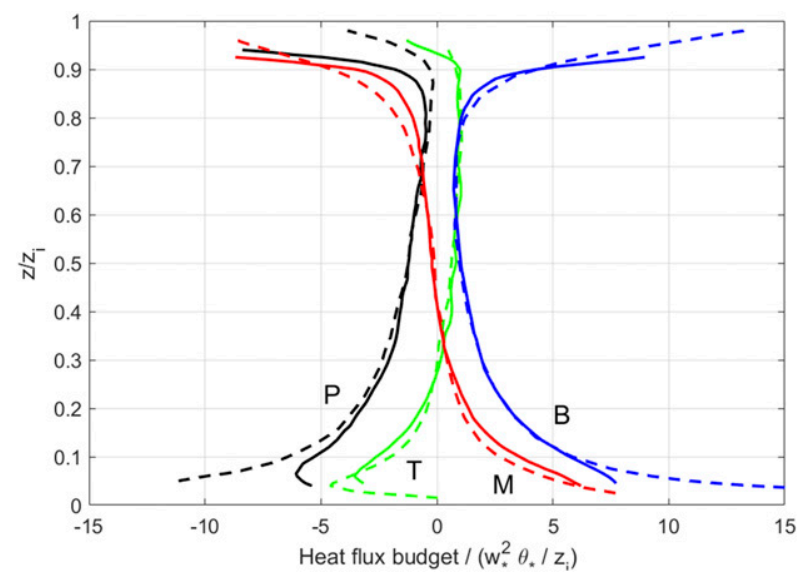

FIG. 1. Vertical profiles of each term of Eq. (1)—P, $T, M$, and $B$-reproduced from Fig. 1 of HM91 (solid lines) and Fig. 5a of GH17 (dashed lines) for strong convection cases.

CBL is generally defined by $0.2<z / z_{i}<0.8$. Also, $w_{*}$ the Deardorff convective velocity scale and $\theta_{*}$ is the convective temperature scale defined as $w_{*} \theta_{*}=w \theta_{s}$, the surface sensible heat flux, that is, the main forcing for pure convection.

LES profiles from HM91 and GH17 are very consistent with each other (Fig. 1) and indicate that the turbulent transport term $T$ cannot be neglected. HM91 proposed $T \approx P+b w_{*}^{2} \theta_{*} / z_{i}$ for $0.1<z / z_{i}<0.8$ where $b \approx 2$ is an empirical value found by HM91 and a modified expression of Eq. (2): $P=-\langle w \theta\rangle / \tau-C_{2} B$ (e.g., Stull 1988, p. 222). Using Moeng and Wyngaard's (1986) results, HM91 used $C_{2}=0.5$, and got an alternative expression for Eq. (4):

$$
\langle w \theta\rangle=-K_{H^{\prime}}\left(\frac{\partial \Theta}{\partial z}-\gamma_{\mathrm{HM}}\right),
$$

with $K_{H^{\prime}}=\left\langle w^{2}\right\rangle \tau / 2$ and

$$
\gamma_{\mathrm{HM}}=b \frac{w_{*}^{2}}{\left\langle w^{2}\right\rangle} \frac{\theta_{*}}{z_{i}} .
$$

Equations (5) and (7) are similar in appearance but are based on different physical processes. Basically, Eq. (5) arises from the buoyancy production term $B$ after neglecting the turbulent transport term $T$. Equation (7) arises from not neglecting $T$ (see HM91, 1691-1692, for more details).

By applying $C_{2}=1 / 3$, expected to be valid for isotropic turbulence (e.g., Stull 1988), a generalized expression of the countergradient term can be written:

$$
\gamma_{\mathrm{G}}=\frac{1}{3} \frac{g}{\theta_{0}} \frac{\left\langle\theta^{2}\right\rangle}{\left\langle w^{2}\right\rangle}+b \frac{w_{*}^{2}}{\left\langle w^{2}\right\rangle} \frac{\theta_{*}}{z_{i}}=\frac{1}{3} \gamma_{\mathrm{D}}+\gamma_{\mathrm{HM}} .
$$




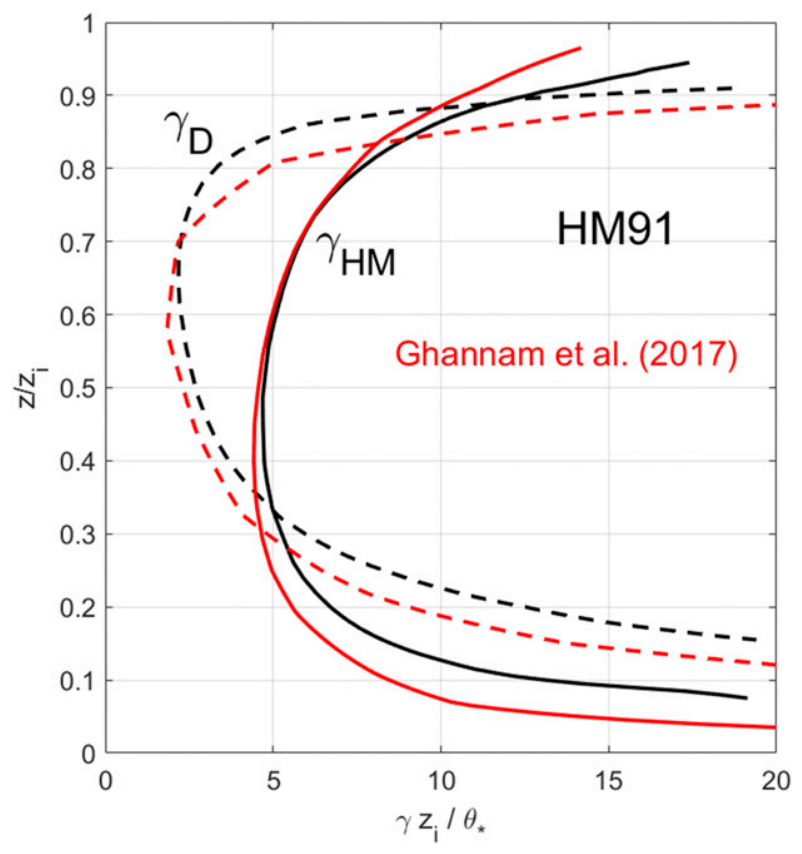

FIG. 2. Vertical profiles of normalized $\gamma_{\mathrm{D}}$ (dashed lines) and $\gamma_{\mathrm{HM}}$ (solid lines) estimated from LES for strong convection according to HM91 (black lines) and GH17 (red lines).

The literature provides many other expressions for the generalized countergradient term, based on various closure schemes and approximations from LES (e.g., Abdella and McFairlane 1997; Tomas 2007; GH17).

Figure 2 shows vertical profiles of $\gamma_{\mathrm{D}}$ and $\gamma_{\mathrm{HM}}$ estimated from LES according to HM91 and calculated from profiles of variances shown by GH17 for the strongly convective case (called S1 in their paper). As noted by HM91, the two expressions of the countergradient term provide a similar behavior but there are substantial differences (e.g., a factor of about 2 around $\left.z / z_{i} \sim 0.7\right)$, which is clearly evident in Fig. 2.

\section{b. Estimation of $\gamma_{D}$ from $C_{T}^{2}$ and $\varepsilon$}

It is difficult to extract the wind velocity, especially its vertical component, accurately from UAV measurements and therefore the variance $\left\langle w^{2}\right\rangle$ cannot be obtained from UAV data. Therefore, Eqs. (5) and (7) for the countergradient term are of little use in our context. However, if we ignore the contribution of anisotropic eddies in the CBL then the ratio of the variances can be rewritten as

$$
\frac{\left\langle\theta^{2}\right\rangle}{\left\langle w^{2}\right\rangle} \approx \frac{C_{\theta}^{2}}{C_{w}^{2}}
$$

where $C_{\theta}^{2}$ and $C_{w}^{2}$ are the structure function parameters for potential temperature and vertical velocity, respectively. Then, we have, from Eq. (5),

$$
\gamma_{D} \approx \frac{g}{\theta_{0}} \frac{C_{\theta}^{2}}{C_{w}^{2}}
$$

Equation (9) was applied by Gossard et al. (1998) for shear generated turbulence in a stably stratified background. However, it might not be indisputably valid in the present case. The main reason is that even if $C_{\theta}^{2}$ and $C_{w}^{2}$ can be defined, Eq. (9) requires that the outer scales of turbulence (identifying the extent of the inertial region of the spectrum) are the same for both the temperature and wind fields, which may or may not be true in the CBL. In addition, observations and LES reported that $\left\langle w^{2}\right\rangle$ slightly differ from $\left\langle\theta^{2}\right\rangle$ or $\left\langle v^{2}\right\rangle$ (with $\left\langle w^{2}\right\rangle \sim 2\left\langle u^{2}\right\rangle$ around the center of the well-mixed portion of the CBL and $\left\langle u^{2}\right\rangle>\left\langle w^{2}\right\rangle$ at the edges) (e.g., Fig. 9 of Moeng and Sullivan 1994) indicating an anisotropic contribution to the variance likely due to the largest convective eddies in the well-mixed portion.

We can estimate $C_{\theta}^{2}$ from $C_{T}^{2}$ as follows, using the relationship between potential temperature and temperature:

$$
C_{\theta}^{2} \approx C_{T}^{2}\left(p_{0} / p\right)^{4 / 7},
$$

where $p_{0}=1000 \mathrm{hPa}$. In the $\mathrm{CBL}, C_{\theta}^{2}$ and $C_{T}^{2}$ are nearly identical. For $z=1000 \mathrm{~m},\left(p_{0} / p\right)^{4 / 7} \approx 1.06$.

On the other hand, $C_{w}^{2}$ reads [Eq. (1b) of Gossard et al. 1998]

$$
C_{w}^{2}=\frac{4}{3} c \varepsilon^{2 / 3},
$$

where $c$ is a universal constant $(\sim 2.1)$. Therefore, a relationship between $\varepsilon$ and $C_{T}^{2}$ can be established:

$$
\gamma_{\mathrm{D}} \approx 0.36 \frac{g}{\theta_{0}} \frac{C_{T}^{2}}{\varepsilon^{2 / 3}},
$$

or equivalently,

$$
\varepsilon=\left(0.36 \frac{g}{\theta_{0}} \frac{1}{\gamma_{\mathrm{D}}} C_{T}^{2}\right)^{3 / 2},
$$

where $0.36=3 /(4 c)$. Equation (14) is the counterpart of Eq. (1) relating $\varepsilon$ and $C_{T}^{2}$ for stratified turbulence. Contrary to Eq. (1), Eq. (14) does not depend on the local static stability $N^{2}$, because the heat flux is dominated by nonlocal transport effects produced by convective eddies in the well-mixed portion of the CBL. Thus, any formulation based only on local gradients would produce incorrect results (see Kantha and Luce 2018).

Now, we are faced with two issues related to Eq. (14). First, this equation was derived from Deardorff's (1966) parameterization of heat flux budget, found later to be 
incorrect by numerical LES studies (HM91). Second, Eq. (13) is conditioned by the validity of Eq. (9). Therefore, we first examined whether Eq. (13) is indeed equivalent to Eq. (5) by comparing with $\gamma_{\mathrm{D}}$ estimated from $\left\langle w^{2}\right\rangle$ and $\left\langle\theta^{2}\right\rangle$. This comparison can be made from published results in the literature, as long as $\varepsilon, C_{T}^{2},\left\langle w^{2}\right\rangle$, and $\left\langle\theta^{2}\right\rangle$ are all estimated. This we do in section 4.

\section{Selection of CBL cases}

The UAV deployment is described by Kantha et al. (2017). The UAVs were usually preprogrammed to move up and down up to a few kilometers above the ground, along spiraling ascents and descents in the vicinity of the $46.5 \mathrm{MHz}$ MU radar (Fukao et al. 1990) and $1.35 \mathrm{GHz}$ LQ7 wind profiler (Imai et al. 2007). Highresolution and low noise wind and temperature data were collected from high-frequency response pitot and cold wire temperature (CWT) sensors, respectively. The data processing used for retrieving pseudovertical profiles of energy dissipation rates and $C_{T}^{2}$ from time series is described in detail by Luce et al. (2019) and there is no need to repeat here. Twenty-six profiles of $\varepsilon$ and $C_{T}^{2}$ (among a total of 66 during CBL events) were selected for this study. The selection procedure was partly subjective but was made in order to retain cases of active, strong CBL and exclude residual layers of a decaying CBL. The selection criteria were based on the following:

1) The depth of the layers $\left(\left\langle z_{i}\right\rangle=1260 \pm 340 \mathrm{~m}\right)$ : The shallow layers of a few hundred meters developing during the early morning just after the sunrise (around $0445 \mathrm{LT})$ were not selected $\left(z_{i}\right.$ is the height of the CBL).

2) The $\varepsilon$ profiles estimated from Pitot data: The selected layers are associated with the largest levels of $\varepsilon$, relatively constant over the depth of the CBL. For $0.2 \leq z / z_{i} \leq 0.8, \log _{10}(\langle\varepsilon\rangle)=-2.50 \pm 0.37$ (i.e., $\langle\varepsilon\rangle \sim 3.2 \mathrm{~mW} \mathrm{~kg}^{-1}$, for the selected cases. By doing so, CBLs in their decaying stage, that is, when the residual layer associated with more variable and significantly smaller values of $\varepsilon$ starts to form, were excluded from the statistics.

3) Independent observations from MU radar (when $z_{i}$ exceeded the altitude of the first radar gate $(\sim 900 \mathrm{~m}$ AGL) and from the LQ7 wind profiler. The morphology of the radar echoes associated with an active CBL is generally easily recognizable. There are bumpy structures of strongly enhanced echo power around the top of the layer, in the capping inversion and entrainment zone. Large vertical velocity disturbances associated with convective updrafts and downdrafts, and enhanced spectral width due to turbulence are clearly evident. The spectral width has been used for estimating kinetic energy dissipation rates from MU radar measurements (Luce et al. 2018). During decaying stages, the radar echoes are significantly weaker around the entrainment zone, and so are the velocity disturbances and spectral width.

4) Humidity profiles, micropulse lidar (MPL) data and fish-eye camera pictures: Sustained convective boundary layers are expected to develop, when radiative heating at the ground is persistent and strong, that is, during periods of clear sky. The absence of clouds in the $\mathrm{CBL}$ and in the free atmosphere was confirmed from MPL data during selected UAV flights. However, sparse broken cumulus clouds were observed at the CBL top during some UAV flights or after (6 flights among 11), suggesting that humid air parcels reached the lifting condensation level on these occasions. Because we did not observe substantial differences between the results with and without clouds at the CBL top, we did not consider the presence of clouds as a relevant factor. Two selected flights were made in presence of midlevel clouds but these flights fulfilled the other conditions (especially number 2).

5) Surface data: The wind speed at the ground was weak (typically about $1-2 \mathrm{~m} \mathrm{~s}^{-1}$ ) during the flights. The solar flux measured at the ground was also useful for estimating the scaling variables.

The time and duration of each of the 11 selected UAV flights are shown in Fig. 3. Each flight provided one to six vertical profiles of atmospheric parameters collected during ascents and descents. The profiles (Fig. 4) associated with the selected flight DH64 (four profiles around $1200 \mathrm{LT}$ ) show the following properties (note that in Figs. 4 and 5, the altitude is MSL and the ground altitude was $348 \mathrm{~m}$ MSL):

1) $N^{2}$ is weak over the whole depth of the CBL layer and shows a pronounced peak at the top, signature of the capping inversion.

2) $C_{T}^{2}$ is strongly enhanced in the $\mathrm{CBL}$ and is maximum around the peak of $N^{2}$. Above $\mathrm{CBL}, C_{T}^{2}$ is about two orders of magnitude weaker. Although not clearly visible on the individual profiles, $C_{T}^{2}$ tends to be minimum near the center of the CBL.

3) $\varepsilon$ is also strongly enhanced in the CBL, but slowly decreases up to the CBL top. Above the CBL top, $\varepsilon$ quickly decreases by almost three orders of magnitude and was too weak to be estimated at some altitude ranges.

These characteristics are at least qualitatively consistent with those reported in the literature for CBL. Section 4 will emphasize the similarities and differences. 


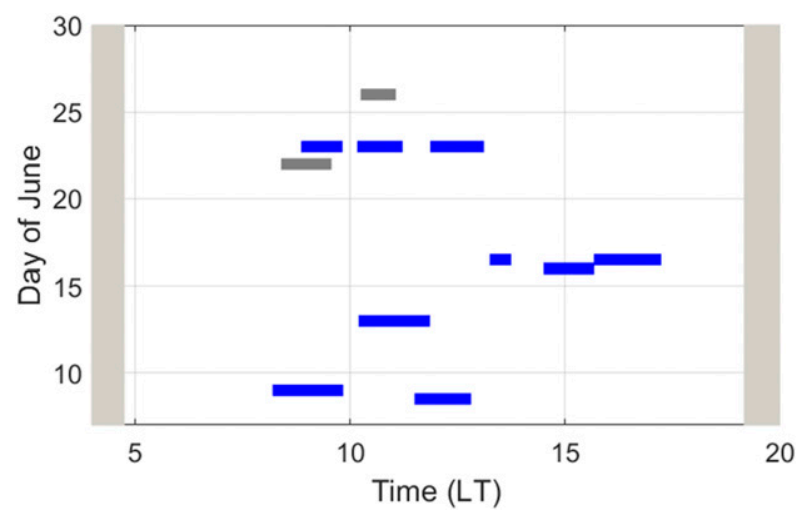

FIG. 3. Time and duration of the 11 UAV flights associated with the selected unsaturated CBLs during clear skies (blue rectangles) and with midlevel clouds (gray rectangles). Sunrise and sunset were around 0445 and 1910 LT, respectively.

Vertical profiles of $N^{2}, C_{T}^{2}$, and $\varepsilon$ for a rejected flight (DH52, $\sim 1500$ LT) are shown in Fig. 5. Contrary to the selected case shown in Fig. 4, enhanced values of $N^{2}$ can be seen around 1000 and $1800 \mathrm{~m}$ MSL below the capping inversion at $2400 \mathrm{~m}$ MSL. The $C_{T}^{2}$ and $\varepsilon$ profiles during DH52 exhibit much weaker values than during DH64 (except around $\sim 1000 \mathrm{~m}$ MSL). They also show large variations with height (up to three orders of magnitude for $\varepsilon$ ) with remarkably similar tendencies between the ascent and descent. These features suggest that a stratified residual layer has started to form. This hypothesis can be strengthened from information provided by the radars (Figs. 6, 7). The time-height cross section of LQ7 radar echo power on 23 June 2017 shows the time evolution of CBLs developing on 20 and 23 June 2017. The enhanced echoes related to CBLs are clearly distinguishable at the bottom of each plot. On 20 June 2017, the echo power was enhanced over the entire CBL until $\sim 1500 \mathrm{LT}$, especially during the three selected UAV flights (DH62, DH63 and DH64). On 23 June 2017, echo power in the CBL started to decrease from $\sim 1400 \mathrm{LT}$ with a more stratified appearance and a deep minimum just below an elevated layer of enhanced echo power generally assumed to be the signature of the CBL top (e.g., Kumar and Jain 2006). The formation of a residual layer, well before the sunset, may be explained by the presence of high-level clouds (decreasing the solar heating and therefore the surface heat flux), followed by precipitation from $\sim 2000$ LT.

Additional information from MU radar observations during DH64 and DH52 consistent with different stages of CBL evolution are shown in Figs. 7a and 7b, respectively. The bumpy structures in the echo power image around the altitude of $2000 \mathrm{~m}$ MSL are the signature of enhanced refractive index turbulence at the edges of the convective cells during DH64 (Fig. 7a). These enhanced
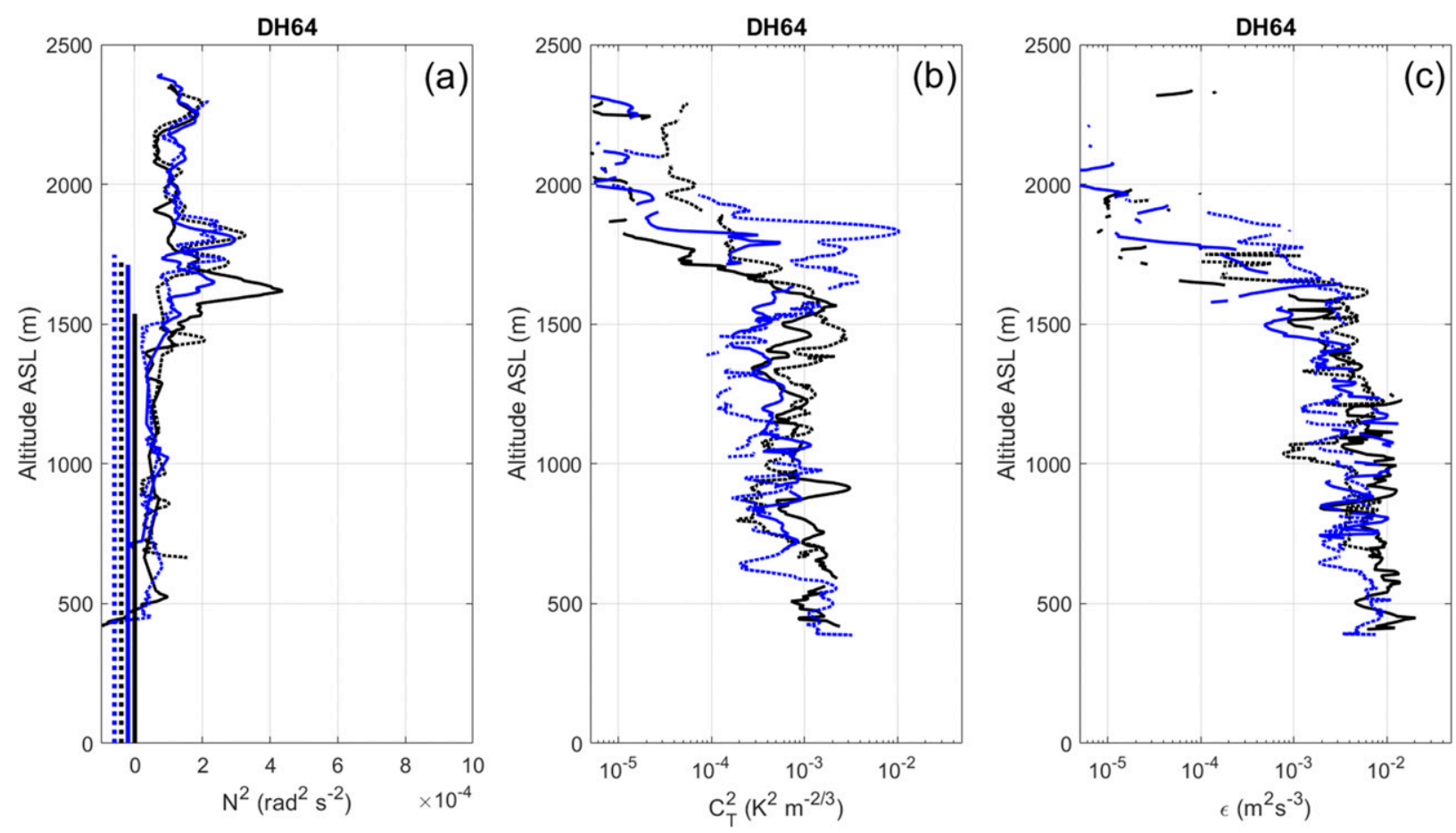

FIG. 4. Example of a selected flight. Vertical profiles of (a) $N^{2}$, (b) $C_{T}^{2}$, and (c) $\varepsilon$ estimated from data gathered during two ascents (black solid and dotted lines) and two descents (blue solid and dotted lines). The vertical lines in (a) indicate the vertical extent of the CBL for each of the four profiles. Note that the altitude is m MSL. 

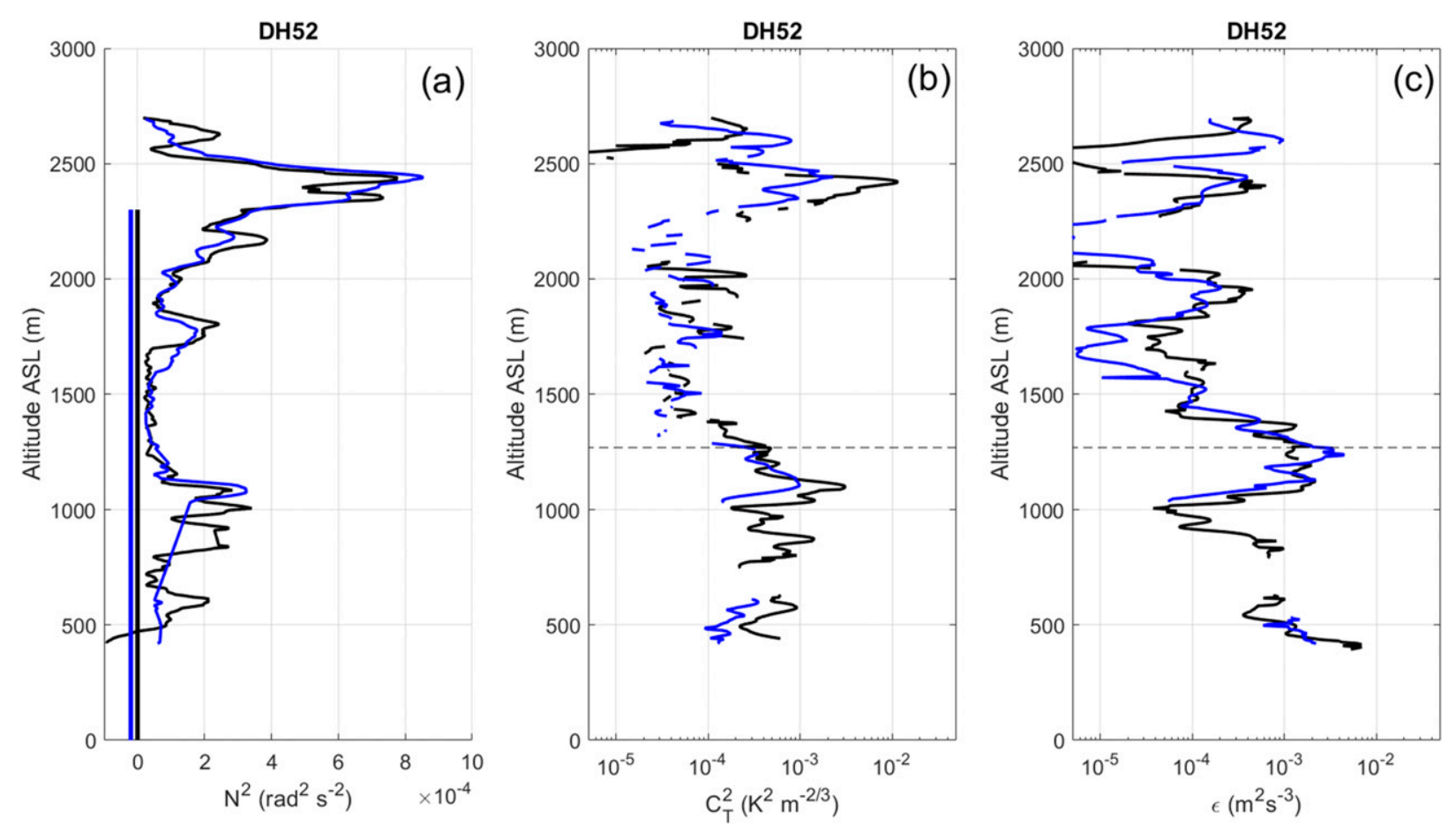

FIG. 5. As in Fig. 4, but for a rejected flight.

echoes clearly delimit the top of a region of enhanced Doppler variance $\sigma^{2}$ due to dynamic turbulence. In addition, strong vertical velocity $W$ fluctuations (exceeding $\pm 2 \mathrm{~m} \mathrm{~s}^{-1}$ ) can be seen, for example, around $1215 \mathrm{LT}$, consistent with strong updrafts and downdrafts in the entrainment zone of the CBL.
In contrast, the radar echo power image for DH52 (Fig. 7b) reveals a nearly flat CBL top at an altitude consistent with the altitude indicated by the LQ7 radar (i.e., $\sim 2500 \mathrm{~m} \mathrm{MSL}$ ). The echo pattern is more typical of stratified conditions. In addition, $\sigma^{2}$ is weakly enhanced within nearly horizontal bands and the $W$ disturbances
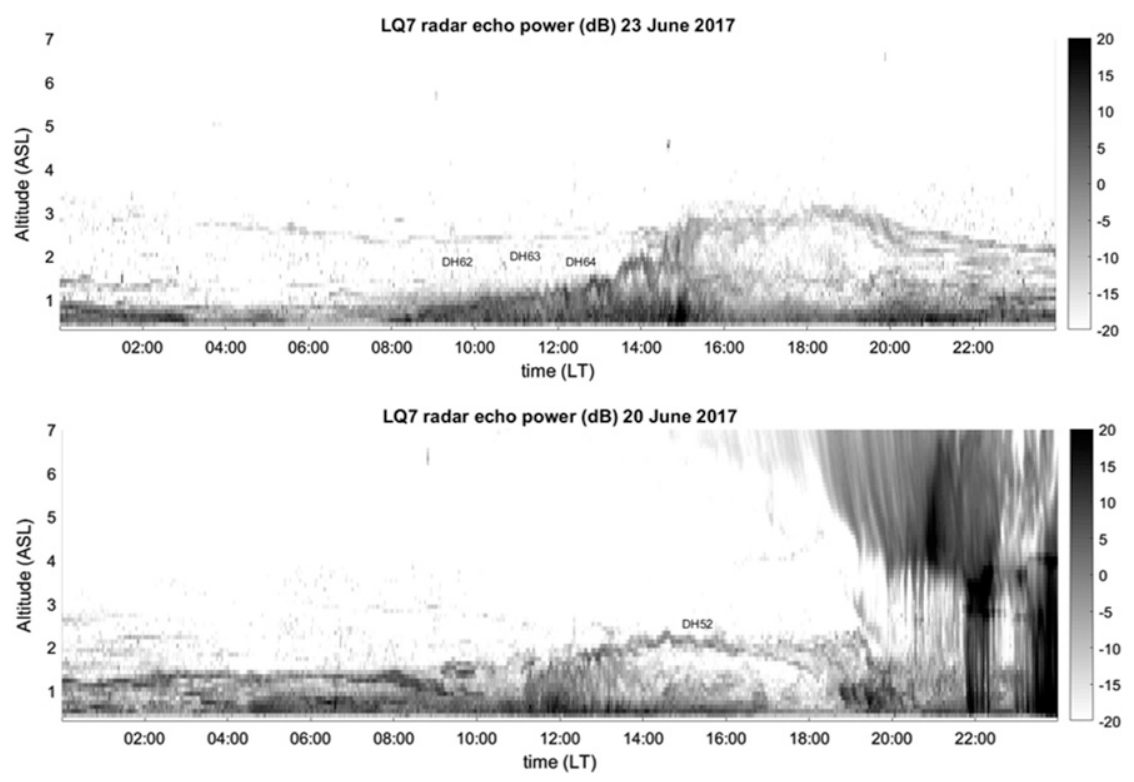

FIG. 6. Time-height cross sections of LQ7 (UHF) radar echo power (dB) at vertical incidence up to the altitude of $7.0 \mathrm{~km}$ on (top) 23 and (bottom) 20 Jun 2017. 

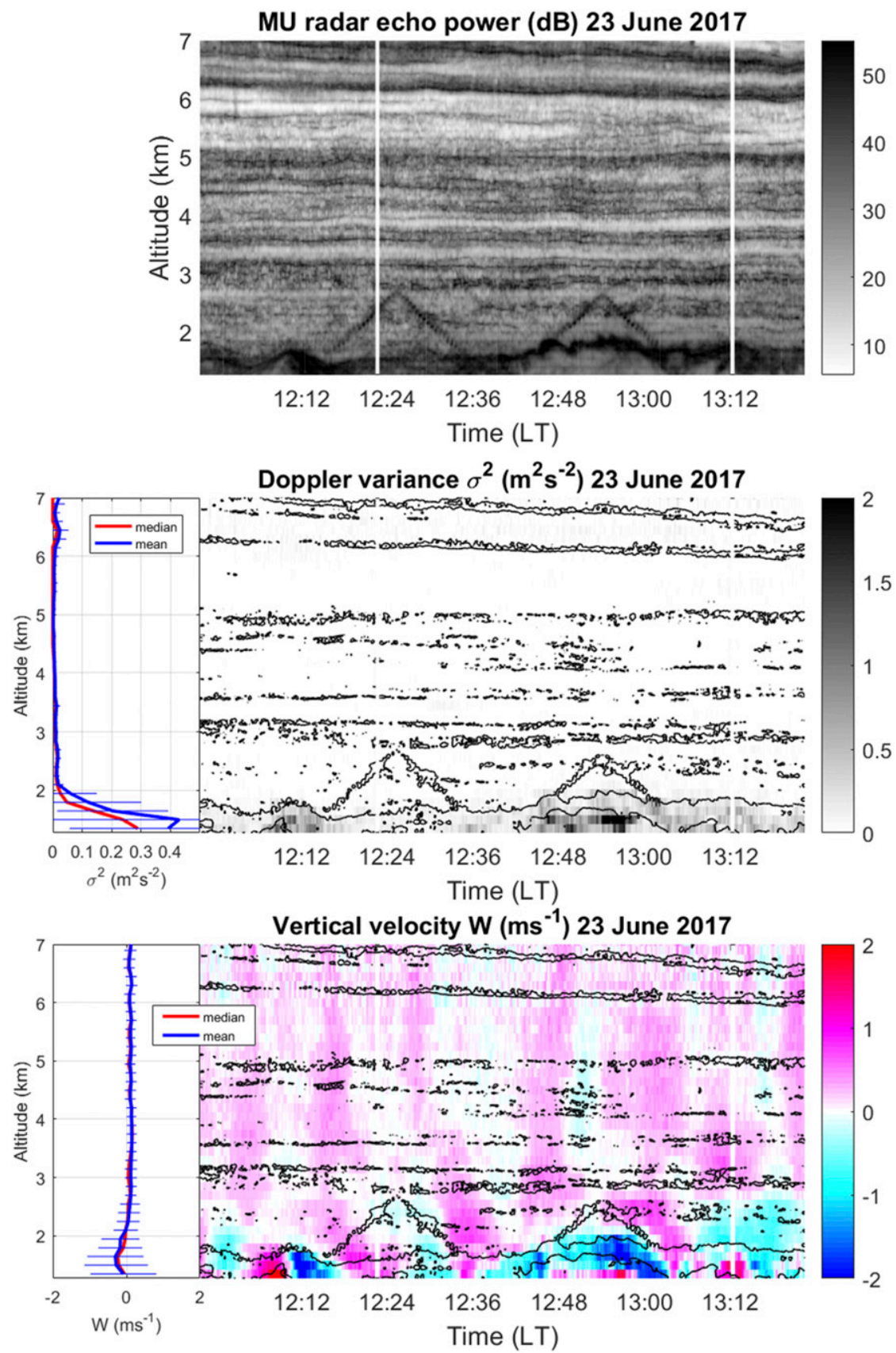

FIG. 7a. Time-height cross sections of (top) MU radar echo power (dB) at vertical incidence operating in range imaging mode on 23 Jun 2017 around DH64 flight, (middle right) Doppler variance $\sigma^{2}\left(\mathrm{~m}^{2} \mathrm{~s}^{-2}\right)$, and (bottom right) vertical velocity $W\left(\mathrm{~m} \mathrm{~s}^{-1}\right)$ up to $7.0 \mathrm{~km}$. Also shown are the mean and median profiles of (middle left) $\sigma^{2}$ and (bottom left) $W$ during the observation window. The horizontal bars indicate \pm 1 standard deviation. For identification of the CBL, the echo power contour level at an arbitrary value is superimposed on the $\sigma^{2}$ and $W$ plots. The triangle-shaped echoes are due to the detection of the UAV. 

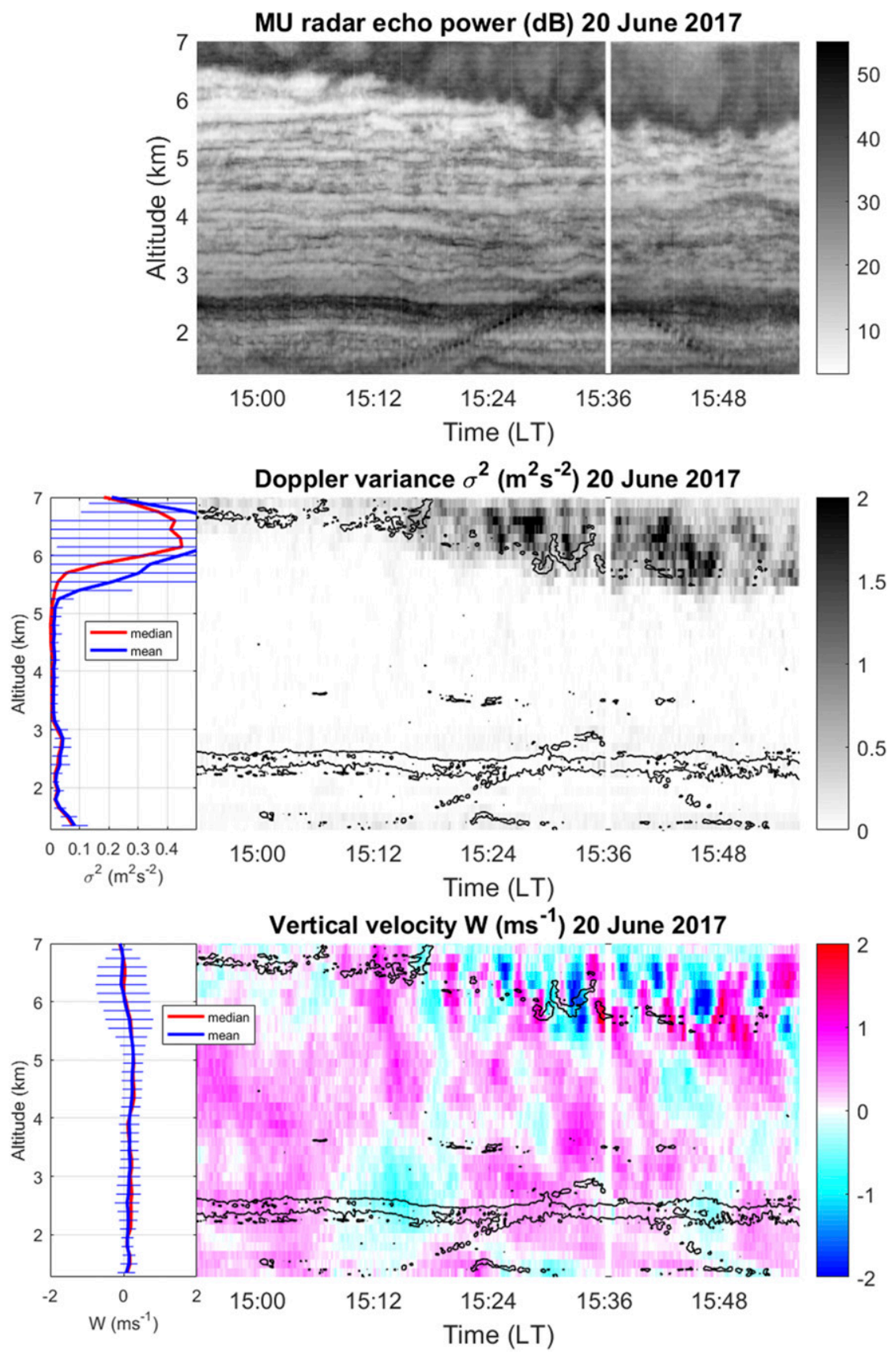

FIG. 7b. As in (a), but for data collected on 20 Jun 2017 around DH52.

are weak. These features confirm the different stages of the above two CBL events.

\section{Results of analyses}

\section{a. Mean profiles of $U A V$-derived parameters} $\left(N^{2}, C_{T}^{2}\right.$, and $\left.\varepsilon\right)$

The 26 selected vertical profiles of $N^{2}, \log _{10}\left(C_{T}^{2} z_{i}^{2 / 3} / \theta_{*}^{2}\right)$, and $\log _{10}\left[\varepsilon /\left(w_{*}^{3} / z_{i}\right)\right]$ versus $z / z_{i}$ are shown in Fig. 8 .
Normalization was made with constant scaling variables $\theta_{*} \approx 0.08 \mathrm{~K}$ and $w_{*} \approx 2 \mathrm{~m} \mathrm{~s}^{-1}$ and $z_{i} \approx 1500 \mathrm{~m}$ making use of surface measurements of solar heating, and coarse approximations for estimating other terms of radiative budget (see appendix 1 of Troen and Mahrt 1986). These scaling values are subject to some uncertainties and produce CBL heights slightly larger than the observed ones, but they are relatively common according to the literature. Part of the scatter in Fig. 8 can be due to normalization with constant 

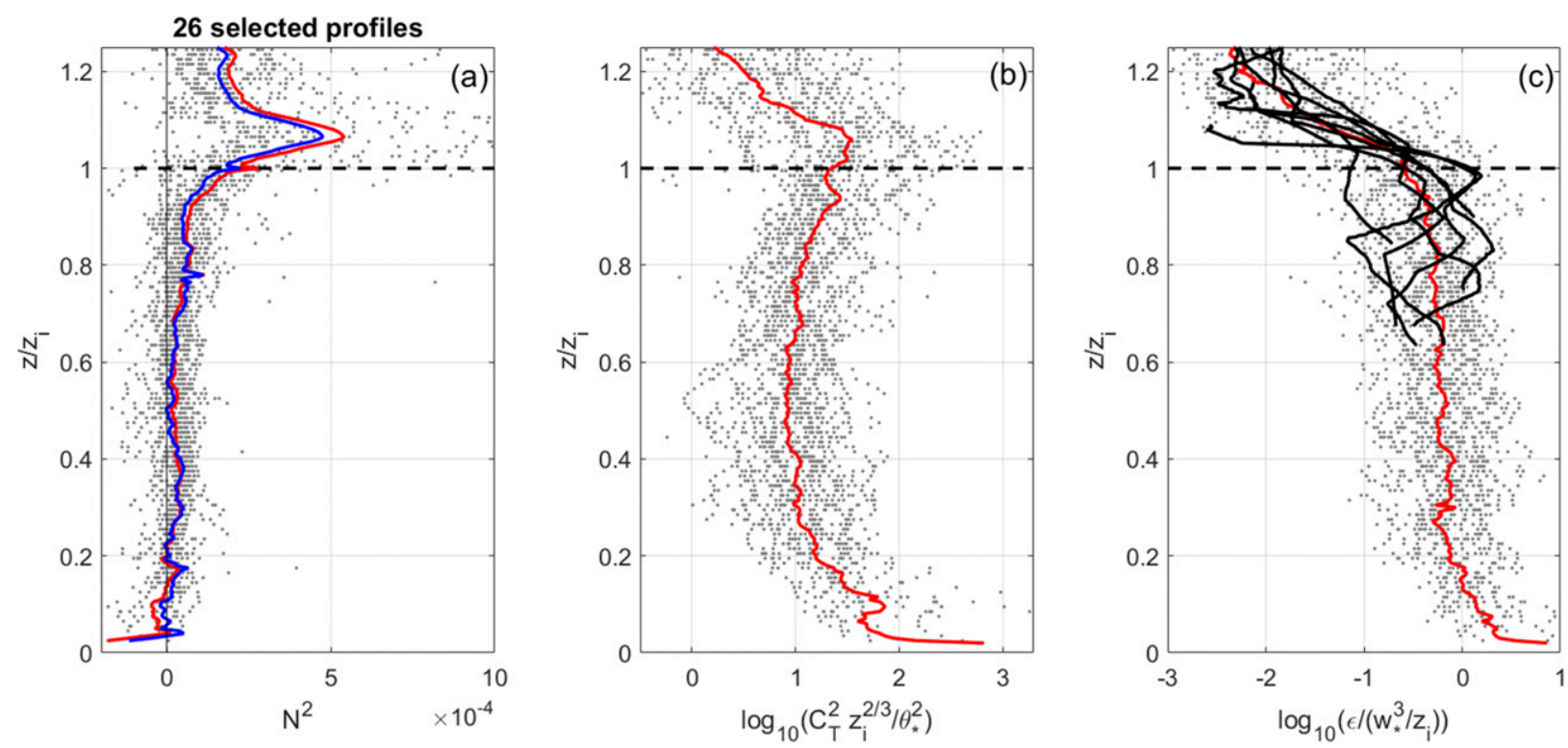

FIG. 8. (a) Vertical profiles of $N^{2}$ estimated from potential temperature, (b) $\log _{10}\left(C_{T}^{2} z_{i}^{2 / 3} / \theta_{*}^{2}\right)$, and (c) $\log _{10}\left[\varepsilon /\left(w_{*}^{3} / z_{i}\right)\right]$ vs $z / z_{i}$ in dotted lines. The red curves show the averaged profiles. In (a), the blue curve shows the mean profile of $N^{2}$ estimated from the virtual potential temperature. In (c), the solid black lines show the corresponding profiles of $\log _{10}\left[\varepsilon /\left(w_{*}^{3} / z_{i}\right)\right]$ estimated from the Doppler variance measured by the MU radar and using the empirical model proposed by Luce et al. (2018). Note that $z$ is the altitude AGL.

values for $\theta_{*}$ and $w_{*}$. However, the mean tendencies with height should not be affected, since more accurate values of $\theta_{*}$ and $w_{*}$ may not be significantly different from the estimated ones. In addition, the comparisons with past observational studies from literature presented in section $4 \mathrm{~b}$ showed that the chosen values make these comparisons quite consistent. Alternative methods used by Kim and Kwon (2019) and based on parameters measured by UAV may be applied for future studies.

The mean profile of $N^{2}$ estimated from the (dry) potential temperature (red curve) is qualitatively consistent with that expected for CBL but shows slightly positive values $\left(\sim 3 \times 10^{-5} \mathrm{rad}^{2} \mathrm{~s}^{-2}\right.$ on average $)$ everywhere in the well-mixed portion of the $\mathrm{CBL}\left(0.2<z / z_{i}<\right.$ $0.8)$. In the standard scheme of a CBL, the mean value of $N^{2}$ is expected to be negative below $z / z_{i} \sim 0.4$ to 0.5 and positive above (e.g., Stull 1988; GH17). Humidity is not the cause of this discrepancy because similar values of $N^{2}$ are obtained from the virtual potential temperature (blue curve) in the well-mixed portion of the CBL. The stability of the capping inversion (above $z / z_{i}>1$ ) is however affected by humidity due the strong decrease of the mixing ratio at the CBL top. At the present time, we do not know if the larger mean values of $N^{2}$ in the well-mixed part of the CBL are due to instrumental or/and atmospheric effects. The $N^{2}$ estimates from UAV measurements can be affected by various biases, especially when vertical air velocities are not negligible with respect to the UAV ascent rate (as may be the case in
CBL; see appendix of Luce et al. 2019). However, concurrent observations from radiosondes showed a similar mean tendency in the CBL (not shown). Also, Bélair et al. (1999) reported potential temperature profiles measured from radiosondes slightly steeper than expected from their numerical simulations (their Fig. 10).

The mean value of $\log _{10}\left(C_{T}^{2} z_{i}^{2 / 3} / \theta_{*}^{2}\right)$ is large near the surface and around the CBL top. It is almost uniform in the well-mixed region near the middle of the CBL but shows a minimum around $z / z_{i} \sim 0.5$, so that the curve is almost symmetric with respect to the center of the CBL. The mean value of $\log _{10}\left[\varepsilon /\left(w_{*}^{3} / z_{i}\right)\right]$ is also maximum near the ground, slowly decreasing with height in the CBL and drops quickly just above $z / z_{i} \sim 1$. Incidentally, profiles of $\log _{10}\left[\varepsilon /\left(w_{*}^{3} / z_{i}\right)\right]$ estimated from MU radar Doppler variances (ShUREX2017) available when CBL was detected above the first altitude sampled by the radar are superimposed (thick solid lines). The processing methods, model and results are described by Luce et al. (2018, 2019). The radar-derived profiles agree quite well with the UAV-derived profiles, partially confirming the conclusions that VHF radars can provide relevant estimates of energy dissipation rates from Doppler spectral width.

\section{b. Comparisons of normalized $\log _{10}\left(C_{T}^{2}\right)$ and $\log _{10}(\varepsilon)$ profiles with literature}

The references used for the comparisons are given in the legends of the figures cited below. Some references 


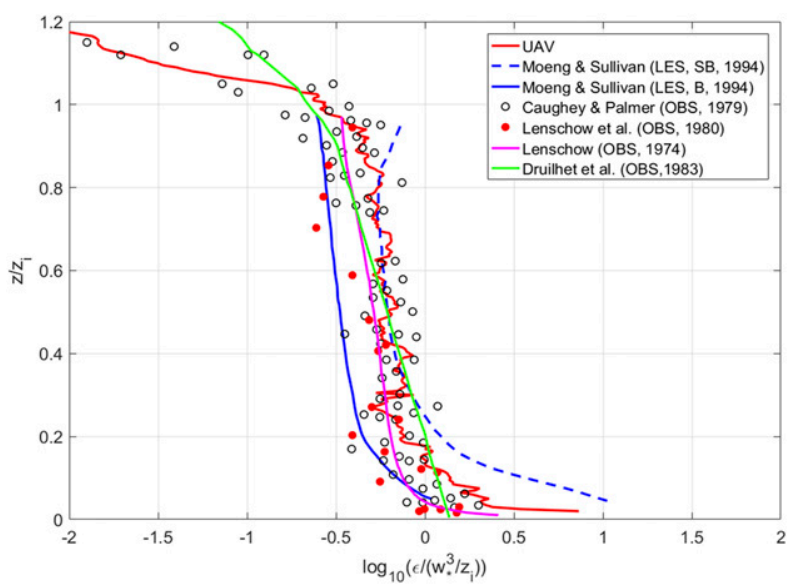

FIG. 9. Vertical profiles of $\log _{10}\left[\varepsilon /\left(w_{*}^{3} / z_{i}\right)\right]$ according to airplane observations and some LES studies superimposed to UAV-derived profiles. The lines and symbols correspond to the references indicated in the legend.

have already applied polynomial fittings for deriving empirical models. When this is not the case, for ease of legibility, some scatterplots have been replaced by a polynomial fit, representative of the tendency of the distributions. Horizontal bars, representative of the variability of these distributions, are not shown, for legibility and because this variability was found sufficiently weak for giving credence to the observed tendency. The reader can find the original plots, sometimes plotted in log scale for altitude, in the indicated references.

Vertical profiles of $\log _{10}\left[\varepsilon /\left(w_{*}^{3} / z_{i}\right)\right]$ derived from UAV data compare well with those derived from in situ measurements (i.e., airplane data) (Caughey and Palmer 1979; Lenschow 1974; Lenschow et al. 1980; Druilhet et al. 1983) and numerical LES (Fig. 9) for strong convection (Moeng and Sullivan 1994). All the dimensionless profiles reveal approximately the same shapes and levels at all altitudes indicating a truly universal tendency with height of the TKE dissipation rate in the CBL.

Vertical profiles of $\log _{10}\left(C_{T}^{2} z_{i}^{2 / 3} / \theta_{*}^{2}\right)$ do not show a consensus in the upper well-mixed portion of the CBL and in the entrainment zone (Fig. 10). In particular, the LES profile from Peltier and Wyngaard (1995) differs strongly from the UAV profile. It has a skewed distribution and exhibits a deep minimum around $z / z_{i} \sim 0.7$. In contrast, the airplane profile from Druilhet et al. (1983) is very similar to the UAV profile and is larger than LES profile by about a factor of $2-3$ around $z / z_{i} \sim 0.7$. The profile described by Caughey and Palmer (1979) has characteristics intermediate to the UAV and LES profiles.

Fairall (1987) has already reported that LES underpredicts $C_{T}^{2}$ in the upper part of the CBL with respect to in situ measurements, suggesting that effects of entrainment might be underestimated by LES. Based on the model of

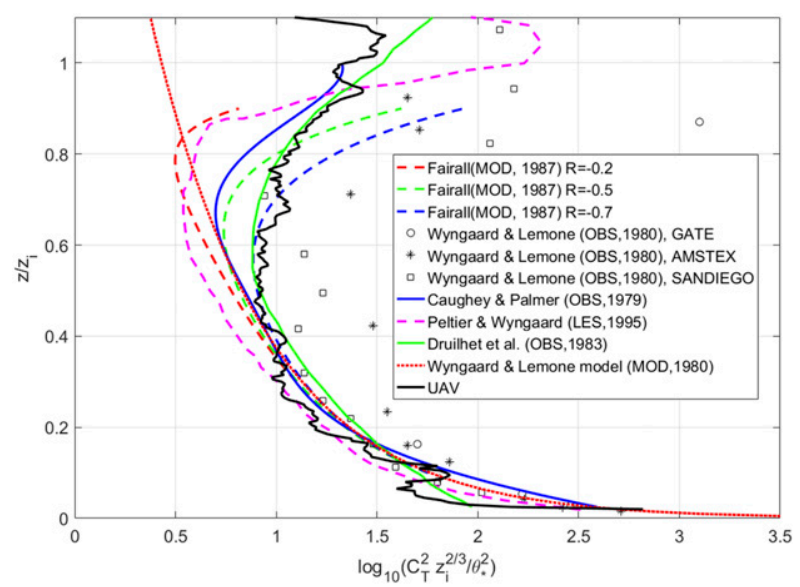

FIG. 10. As in Fig. 9, but for $\log _{10}\left(C_{T}^{2} z_{i}^{2 / 3} / \theta_{*}^{2}\right)$. The lines and symbols correspond to the references indicated in the legend.

top-down and bottom-up diffusion introduced by Moeng and Wyngaard (1986) and Fairall (1987) used an additional scaling parameter $R$ defined as the ratio of entrainment (top) heat flux to the surface heat flux, for modeling entrainment effects. The value of $R$ is $\sim-0.2$ for conventional LES of CBL (strong convection). For this value, the LES profile is consistent with the Fairall model (dashed red and pink curves of Fig. 10). For smaller (more negative) values of $R(-0.5,-0.7), C_{T}^{2}$ significantly increases in the upper part of the CBL and the altitude of the minimum of $C_{T}^{2}$ decreases, but these profiles do not fit the observed ones (except Caughey and Palmer's profile, to some extent, for $R=-0.5$ ). Observations from Wyngaard and Lemone (1980) show a large dispersion and do not seem to be consistent with any of the other profiles. Therefore, they are not considered for the rest of this work. The domain of validity of the Wyngaard and Lemone model (red dotted line) does not extend above the well-mixed part of the CBL (i.e., $z / z_{i}<0.8$ ).

\section{c. Comparisons between profiles of $\left\langle w^{2}\right\rangle$ and $\left\langle\theta^{2}\right\rangle$}

As for $\varepsilon$, the vertical profiles of normalized vertical velocity variance $\left\langle w^{2}\right\rangle_{n}=\left\langle w^{2}\right\rangle / w_{*}^{2}$ in the CBL obtained from LES, models or airplane observations do not show significant differences, except near the surface (Fig. 11). In contrast, the vertical profiles of normalized $\left\langle\theta^{2}\right\rangle_{n}=\left\langle\theta^{2}\right\rangle / \theta_{*}^{2}$ from LES studies (HM91; Tomas 2007; GH17) clearly differ from observed $\left\langle\theta^{2}\right\rangle_{n}$ (Caughey and Palmer 1979; Lenschow et al. 1980; Druilhet et al. 1983; Therry and Lacarrère 1983) (Fig. 12). All the LES $\left\langle\theta^{2}\right\rangle_{n}$ profiles for strong convection show a trend similar to normalized $C_{T}^{2}, C_{T n}^{2}$ LES profiles, that is, a deep minimum around $z / z_{i} \sim 0.7$. All the observed $\left\langle\theta^{2}\right\rangle_{n}$ profiles show larger values, especially near the entrainment zone, but observed $\left\langle\theta^{2}\right\rangle_{n}$ values converge toward 


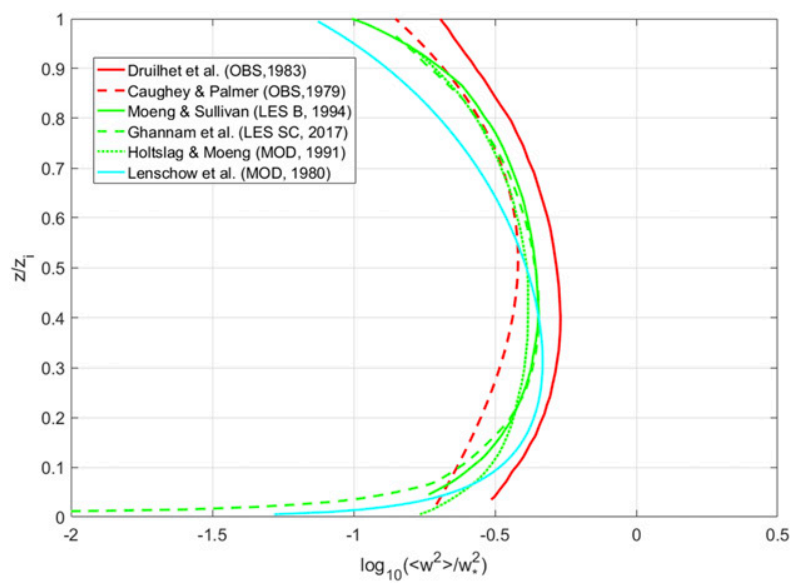

FIG. 11. As in Fig. 9, but for $\log _{10}\left(\left\langle w^{2}\right\rangle_{n}\right)$. The lines are associated with references indicated in the legend.

LES $\left\langle\theta^{2}\right\rangle_{n}$ values near the top and bottom of the CBL. As a result, the observed $\left\langle\theta^{2}\right\rangle_{n}$ profiles show a lower variability with height. The mean LES $\left\langle\theta^{2}\right\rangle_{n}$ (blue curve) and observed $\left\langle\theta^{2}\right\rangle_{n}$ (pink curve) profiles vary by over a factor of 5 and 2, respectively, in the well-mixed part of the CBL $\left(0.2<z / z_{i}<0.8\right)$. However, LES results do seem to provide better agreement with laboratory experiments reported by Willis and Deardorff (1974).

Comparisons between the mean observed $\left\langle\theta^{2}\right\rangle_{n}$, LES $\left\langle\theta^{2}\right\rangle_{n}$, and model $\left\langle\theta^{2}\right\rangle_{n}$ profiles obtained for strong, moderate, and weak convection are shown in Fig. 13. The various LES and models agree very well with one another for each of the convection intensity, but none of the LES and model profiles fit the mean observed profile. This indicates that the discrepancies between LES/models and observations cannot simply be explained away by improper scaling, and that some other issue exists. The validation of Eq. (14) is thus made difficult by these substantial differences between observations and numerical simulations.

\section{d. Attempt at validation of $\gamma_{D}$ from Eq. (13)}

To the authors' knowledge, Caughey and Palmer (1979) (Figs. 4, 5, 9, 10) and Druilhet et al. (1983) (Figs. 3,4$)$ are the sole studies providing profiles of (normalized) $\varepsilon, C_{T}^{2},\left\langle w^{2}\right\rangle$, and $\left\langle\theta^{2}\right\rangle$ from the same datasets. Profiles of $\gamma_{\mathrm{D} n}=\gamma_{\mathrm{D}} z_{i} / \theta_{*}$ given by Eqs. (5) and (14) are shown in Fig. 14, along with the UAV profile derived from the mean values of $\varepsilon, C_{T}^{2}$ shown in Fig. 8 . The profiles obtained from Caughey and Palmer's data yield similar results for $z / z_{i}<0.3$ but the two profiles diverge at higher altitudes: $\gamma_{\mathrm{D} n}$ values from Eq. (13) exceed the normalized values from Eq. (5) by a factor of 3 in the entrainment zone $\left(z / z_{i}>0.8\right)$. However, the two profiles obtained from Druilhet

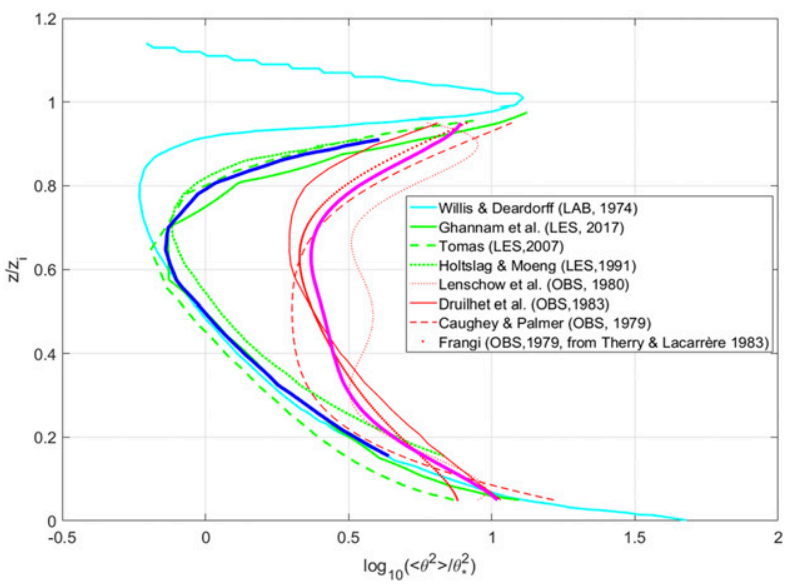

FIG. 12. As in Fig. 9, but for $\log _{10}\left(\left\langle\theta^{2}\right\rangle_{n}\right)$. The lines are associated with references indicated in the legend.

et al.'s data are very similar, giving some credence to the theoretical derivations proposed in section $2 \mathrm{~b}$. In addition, they coincide very well with the UAV profile indicating that the CBL cases studied by Druilhet et al. (1983) were likely comparable with those we observed, and not Caughey and Palmer (1979). The reason for this discrepancy is not known.

The profiles of LES $\gamma_{\mathrm{D} n}$ and $\gamma_{\mathrm{HM} n}$ (Fig. 2) are superimposed on the profiles of observed $\gamma_{\mathrm{D} n}$ (Fig. 14b) in Fig. 15. One apparent paradox stands out: LES $\gamma_{\mathrm{HM} n}$ (solid blue) fits observed $\gamma_{\mathrm{D} n}$ (black, green, and red curves) much more accurately than LES $\gamma_{\mathrm{D} n}$ (dotted blue). The poor agreement between observed $\gamma_{\mathrm{D} n}$ and LES $\gamma_{\mathrm{D} n}$ was expected due to the differences between observed $\left\langle\theta^{2}\right\rangle_{n}$ and LES $\left\langle\theta^{2}\right\rangle_{n}$ around $z / z_{i} \sim 0.7$ (see Fig. 12). The good agreement between LES $\gamma_{\mathrm{HM} n}$ and observed $\gamma_{\mathrm{D} n}$ is more puzzling, but it may suggest that the two counter gradient terms $\gamma_{\mathrm{HM}}$ and $\gamma_{\mathrm{D}}$ are in practice quantitatively similar, despite different physical mechanisms. Since LES $\left\langle w^{2}\right\rangle_{n}$ and observed $\left\langle w^{2}\right\rangle_{n}$ do not differ substantially (see Fig. 11), LES $\gamma_{\mathrm{HM} n}$ also fit observed $\gamma_{\mathrm{HM} n}$.

The paradox may be tentatively resolved from the following arguments:

$\gamma_{\mathrm{HM} n}$ can be rewritten as

$$
\gamma_{\mathrm{HM} n}=\frac{2}{\left\langle w^{2}\right\rangle_{n}} .
$$

Also,

$$
\gamma_{\mathrm{D} n}(\mathrm{OBS}) \approx\left[\frac{g}{\theta_{0}}\left\langle\theta^{2}\right\rangle_{n}(\mathrm{OBS}) \frac{\theta_{*} z_{i}}{w_{*}^{2}}\right] \frac{1}{\left\langle w^{2}\right\rangle_{n}} .
$$

Equations (15) and (16) are equivalent if the term in the square brackets in (16) can be approximated by a 


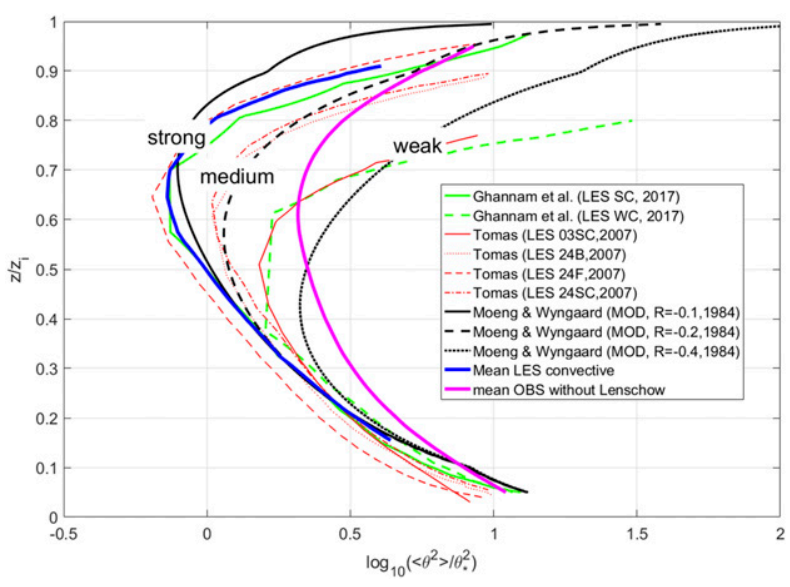

FIG. 13. Profiles of $\log _{10}\left(\left\langle\theta^{2}\right\rangle_{n}\right)$ for various intensities of convection according to LES and models. The lines correspond to references indicated in the legend.

constant equal to 2. As mentioned earlier, observed $\left\langle\theta^{2}\right\rangle_{n}$ varies over a factor of 2 only in the well-mixed part of the CBL $\left(0.2<z / z_{i}<0.8\right)$ (see Fig. 12). Its mean value is about $2.8\left(0.45 \mathrm{in} \log _{10}\right.$ scale $)$. Thus,

$$
\gamma_{\mathrm{D} n}(\mathrm{OBS}) \approx 2.3 \frac{1}{\left\langle w^{2}\right\rangle_{n}} \approx 1.15 \gamma_{\mathrm{HM} n}(\mathrm{LES})
$$

for the mean values of $\theta_{*}, w_{*}$, and $z_{i}$ used in the present study. The generalized expression (8) would read observed $\gamma_{\mathrm{G} n} \approx 1.2 \gamma_{\mathrm{D} n}$. The numerical coefficients strongly

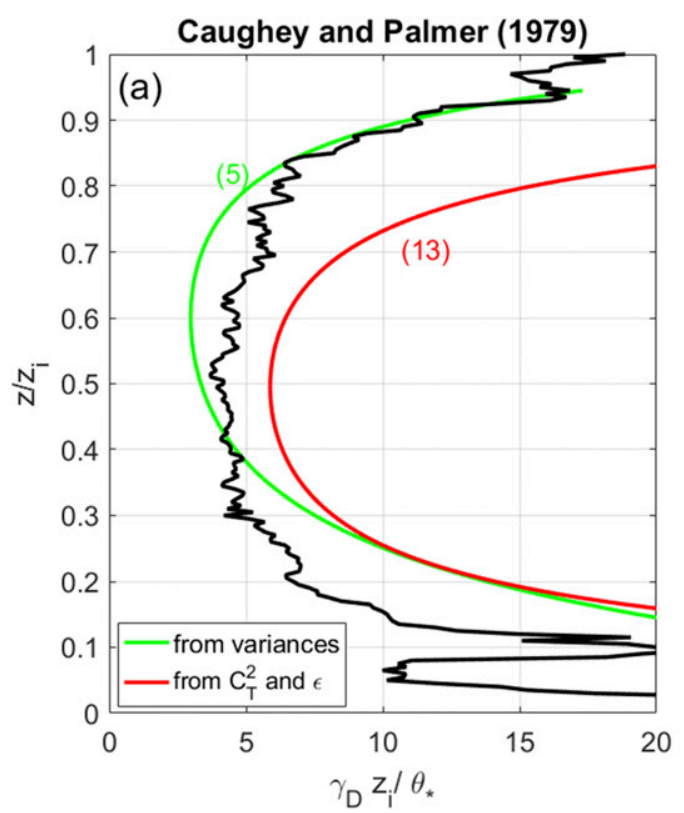

depend on the values of the scaling variables, but observed $\gamma_{\mathrm{D} n}$ and $\gamma_{\mathrm{G} n}$ would nearly be proportional to LES $\gamma_{\mathrm{HM} n}$, in any case.

These derivations are relevant only if the Eq. (15) for $\gamma_{\mathrm{HM} n}$, based on closure schemes recalled in section $2 \mathrm{a}$, is still valid despite changes of $\left\langle\theta^{2}\right\rangle_{n}$, that is, changes of the buoyancy production term $B$ of the heat flux budget. An increase of the term $B$ must imply a decrease of $(M+P+T)$ [see Eq. (1)] for equilibrium. The closure expression used by HM91 for the term $T$ may not be valid anymore. Interestingly, a decrease of $T$ above $z / z_{i}>0.3$ tends to make it closer to 0 , which would be in agreement with the hypothesis made by Deardorff (1966) for deriving Eq. (5). A decrease of $M$ without changes of $\left\langle w^{2}\right\rangle$ implies an increase of $d \Theta / d z$ (or $N^{2}$ ) with respect to the level expected from LES. It is at least qualitatively consistent with what we observed in Fig. 8a. These assertions are, of course, rather speculative and require additional in situ and LES studies for validation.

\section{e. Application of models of countergradient terms}

The mean profiles of normalized $\varepsilon$ (in log scale) reconstructed from $C_{T}^{2}$ using Eq. (14) and various expressions of the countergradient terms,

$$
\gamma_{\mathrm{D}}(\mathrm{LES})=\frac{g}{\theta_{0}} \frac{\left\langle\theta^{2}\right\rangle_{\mathrm{LES}}}{\left\langle w^{2}\right\rangle_{\mathrm{LES}}},
$$

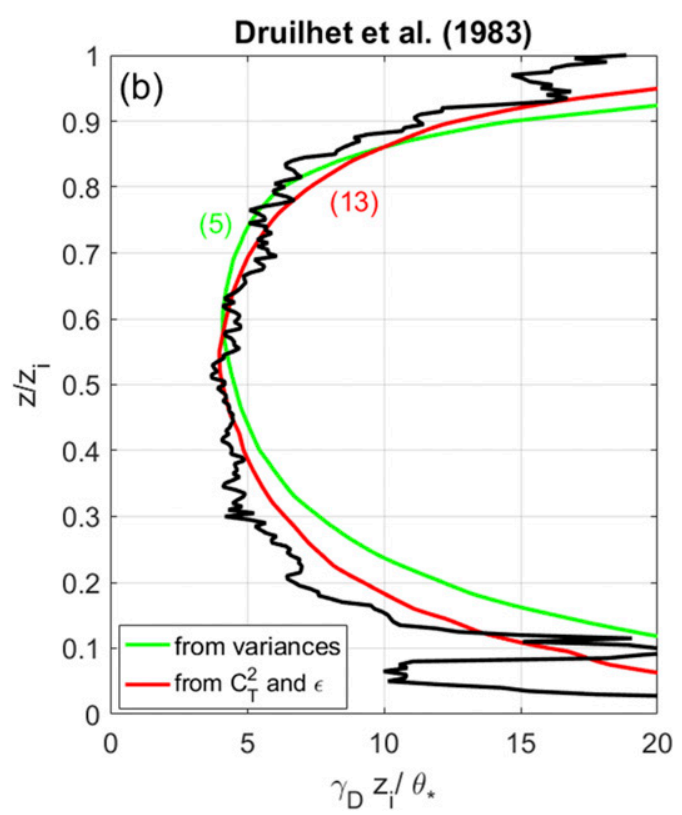

FIG. 14. Vertical profiles of $\gamma_{\mathrm{D} n}=\gamma_{\mathrm{D}} z_{i} / \theta_{*}$ from Eq. (5) (green curve) and Eq. (13) (red curve) from data in (a) Caughey and Palmer (1979) and (b) Druilhet et al. (1983). The black curve shows the corresponding estimates from UAV data using Eq. (13). 


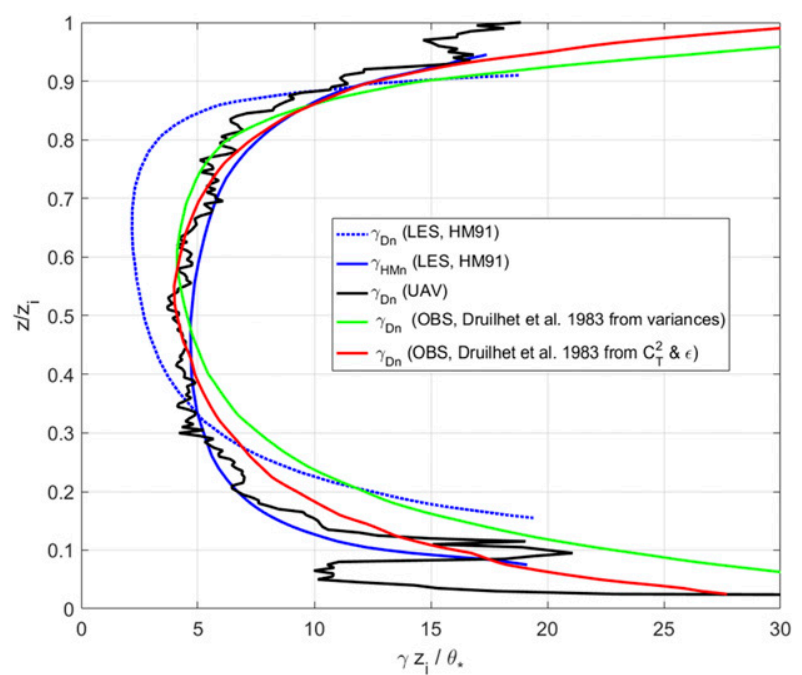

FIG. 15. Vertical profiles of $\gamma_{\mathrm{D} n}(\mathrm{LES})$ and $\gamma_{\mathrm{HM} n}(\mathrm{LES})$ performed by HM91 (blue curve). The black, green, and red curves show the normalized $\gamma_{\mathrm{D} n}(\mathrm{OBS})$ estimated from UAV and Druilhet et al. (1983) data (as in Fig. 13).

$$
\begin{aligned}
\gamma_{\mathrm{HM}}(\mathrm{LES}) & =b \frac{w_{*}^{2}}{\left\langle w^{2}\right\rangle_{\mathrm{LES}}} \frac{\theta_{*}}{z_{i}}, \\
\gamma_{\mathrm{D}}(\mathrm{Dr} 83) & =\frac{g}{\theta_{0}} \frac{\left\langle\theta^{2}\right\rangle_{\mathrm{Dr} 83}}{\left\langle w^{2}\right\rangle_{\mathrm{Dr} 83}}
\end{aligned}
$$

are compared with measured profiles in Fig. 16. In Eq. (18c) $\gamma_{\mathrm{D}}($ Dr83) is the Deardorff countergradient term estimated from variances provided by Druilhet et al. (1983). As expected, the reconstructed profiles from $\gamma_{\mathrm{D}}$ (LES) agree poorly with the measured profiles. In contrast, the agreement is satisfying when using $\gamma_{\mathrm{HM}}$ (LES) or $\gamma_{\mathrm{D}}(\mathrm{Dr} 83)$. The absolute level of the countergradient term would be about $\gamma_{\mathrm{D}} \sim 0.21 \times 10^{-3}$ to $0.37 \times$ $10^{-3} \mathrm{Km}^{-1}$ in the well-mixed region where it is roughly constant. These values compare quite well with the values reported by Deardorff $(1972)\left(\gamma_{\mathrm{D}} \sim 0.31 \times 10^{-3}\right.$ to $0.52 \times 10^{-3} \mathrm{Km}^{-1}$ ) from airplane observations.

\section{Summary and conclusions}

We have proposed a theoretical expression [Eq. (14)] relating the TKE dissipation rate $\varepsilon$ to $C_{T}^{2}$ in a nonsaturated CBL, based on Deardorff's (1972) formulation [Eq. (5)] of the countergradient term $\gamma_{\mathrm{D}}$ for sensible heat flux (section 2). This expression, expected to be valid in the well-mixed central region of the $\mathrm{CBL}$ within the framework of Deardorff's hypotheses, is $\varepsilon=\alpha\left(C_{T}^{2}\right)^{3 / 2}$, where $\alpha=\left(0.36 g / \theta_{0} \gamma_{\mathrm{D}}\right)^{3 / 2}$. It is the counterpart of Eq. (1) valid for stably stratified turbulence only. We investigated Eq. (14) by evaluating $\gamma_{\mathrm{D}}$ from observational and LES data available in the literature and by considering an alternative expression for the countergradient term $\gamma_{\mathrm{HM}}$ from HM91, which was based on a different physical argument. These investigations have led to a cascade of puzzling results, which we summarize below:

1) The normalized profile of $\gamma_{D}$ estimated from Eq. (5) and using airplane-derived $\theta^{2}$ and $w^{2}$ profiles reported by Druilhet et al. (1983) is similar to the one estimated from Eq. (13) using $\mathrm{UAV}$-derived $\varepsilon$ and $C_{T}^{2}$ (Fig. 14a). The agreement when using Caughey and Palmer (1979) aircraft data is worse (Fig. 14b), but the results suggest that Eq. (14) is quantitatively satisfactory, although the isotropy hypothesis applied to derive Eq. (13) may be disputable. Therefore, these first results would make the problem relatively easily solved, but the Deardorff formulation was questioned by subsequent studies using LES (e.g., HM91).

2) We further found that the profiles of $\gamma_{D}$ estimated from LES using Eq. (5) do not fit those estimated from airplanes and UAV. The cause of this discrepancy is the significant difference (up to a factor of 3) between normalized LES-derived, and $\left\langle\theta^{2}\right\rangle$ (and $C_{T}^{2}$ ) values observed in the upper part of the well-mixed portion of the CBL. There is no such difference between LES-derived and observed $\left\langle w^{2}\right\rangle$ values.

3) By contrast, the countergradient term $\gamma_{\mathrm{HM}}$ estimated from LES differs from $\gamma_{\mathrm{D}}$ estimated from LES (Fig. 2), but confusingly fits $\gamma_{\mathrm{D}}$ estimated from UAV and airplane data (Fig. 15). This puzzling agreement can be explained only if $\left\langle\theta^{2}\right\rangle$ is relatively constant in the wellmixed portion of the CBL, because $\gamma_{\mathrm{D}}$ and $\gamma_{\mathrm{HM}}$ are proportional to $\left\langle\theta^{2}\right\rangle /\left\langle w^{2}\right\rangle$ and $1 /\left\langle w^{2}\right\rangle$, respectively. The LES-derived $\left\langle\theta^{2}\right\rangle$ does not respect this condition but, to some extent, airplane-derived $\left\langle\theta^{2}\right\rangle$ does. Therefore, based on observed $\left\langle\theta^{2}\right\rangle$, we found that $\gamma_{\mathrm{D}}$ and $\gamma_{\mathrm{HM}}$ would be virtually identical, at least in shape, despite different physical hypotheses made in their derivations. The more general expression [Eq. (9)], combining $\gamma_{\mathrm{D}}$ and $\gamma_{\mathrm{HM}}$ would also yield similar profiles.

4) It turns out that if the observed $\left\langle\theta^{2}\right\rangle$ values are more representative than the LES-derived $\left\langle\theta^{2}\right\rangle$ the heat flux budget must be reexamined, since $\left\langle\theta^{2}\right\rangle$ contributes to the buoyancy production term $B$. Therefore, turbulence closure schemes used for deriving $\gamma_{\mathrm{HM}}$ may not be valid anymore. It is clear that we have fallen into a circular reasoning. It is possible to avoid it, only if the causes of disagreement between LES and observations are explained as being due to either the inadequacy of LES to model the complex mechanisms of entrainment at and near the CBL top (often recognized as much deeper down to the well-mixed 

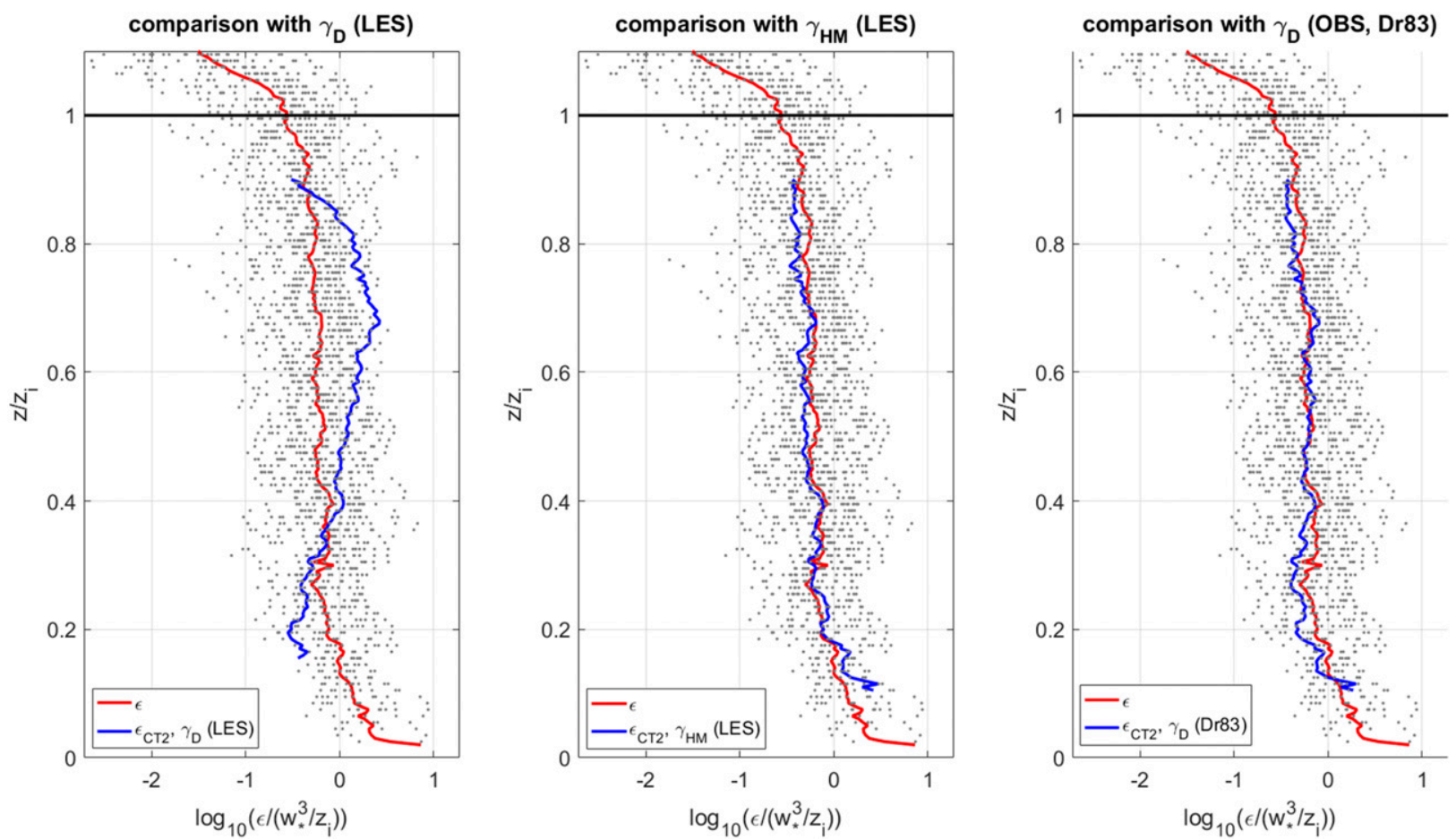

FIG. 16. Vertical profiles of $\log _{10}\left[\varepsilon /\left(w_{*}^{3} / z_{i}\right)\right]$ (dots and red curve for the average; Fig. 8) superimposed on the reconstructed profiles from $C_{T}^{2}$ using Eq. (13) for various expressions [Eqs. (18a)-(18c)] of the countergradient term.

portion of the $\mathrm{CBL}$ ) or measurement biases from in situ techniques. In addition, the present analysis focused on mean tendencies. The analysis of each individual profile might yield other insights, to be considered in future work on the topic.

Acknowledgments. The authors thank T. Mixa, R. Wilson, and T. Tsuda for their cooperation during the campaigns. This study was partially supported by JSPS KAKENHI Grant JP15K13568 and the research grant for Mission Research on Sustainable Humanosphere from Research Institute for Sustainable Humanosphere (RISH), Kyoto University. Partial support to DL was provided by NSF Grant Instabilities, Dynamics and Energetics accompanying Atmospheric Layering (IDEAL) AGS 104163

\section{REFERENCES}

Abdella, K., and N. McFairlane, 1997: A new second-order turbulence closure scheme for the planetary boundary layer. J. Atmos. Sci., 54, 1850-1867, https://doi.org/10.1175/15200469(1997)054<1850:ANSOTC>2.0.CO;2.

Bélair, S., J. Mailhot, J. W. Strapp, and J. I. MacPherson, 1999: An examination of local versus nonlocal aspects of a THEbased boundary-layer scheme in clear convective conditions. J. Appl. Meteor., 38, 1499-1518, https://doi.org/ 10.1175/1520-0450(1999)038<1499:AEOLVN>2.0.CO;2.
Caughey, S. J., and S. G. Palmer, 1979: Some aspects of turbulence structure through the depth of the convective boundary layer. Quart. J. Roy. Meteor. Soc., 105, 811-827, https://doi.org/ 10.1002/qj.49710544606.

Deardorff, J. W., 1966: The counter-gradient heat flux in the lower atmosphere and in the laboratory. J. Atmos. Sci., 23, 503-506, https://doi.org/10.1175/1520-0469(1966)023<0503:TCGHFI> 2.0.CO;2.

1972: Theoretical expression for the counter-gradient vertical heat flux. J. Geophys. Res., 77, 5900-5904, https://doi.org/ 10.1029/JC077i030p05900.

Druilhet, A., J. P. Frangi, D. Guédalia, and J. Fontan, 1983: Experimental studies of the turbulence structure parameters of the convective boundary layer. J. Climate Appl. Meteor., 22, 594-608, https://doi.org/10.1175/1520-0450(1983)022<0594: ESOTTS $>2.0$.CO;2.

Fairall, C. W., 1987: A top-down and bottom-up diffusion model of $C_{T}^{2}$ and $C_{Q}^{2}$ in the entraining convective boundary layer. J. Atmos. Sci., 44, 1009-1017, https://doi.org/10.1175/15200469(1987)044<1009:ATDABU>2.0.CO;2.

Fukao, S., T. Sato, T. Tsuda, M. Yamamoto, M. D. Yamanaka, and S. Kato, 1990: MU radar: New capabilities and system calibrations. Radio Sci., 25, 477-485, https://doi.org/10.1029/ RS025i004p00477.

Ghannam, K., T. Dunam, S. T. Salesky, M. Chamecki, and G. Katul, 2017: The non-local character of turbulence asymmetry in the convective atmospheric boundary layer. Quart. J. Roy. Meteor. Soc., 143, 494-507, https://doi.org/10.1002/ qj.2937.

Gossard, E. E., D. E. Wolfe, K. P. Moran, R. A. Paulus, K. D. Anderson, and L. T. Rogers, 1998: Measurement of clear-air gradients and turbulence properties with radar wind profilers. 
J. Atmos. Oceanic Technol., 15, 321-342, https://doi.org/ 10.1175/1520-0426(1998)015<0321:MOCAGA >2.0.CO;2.

Holtslag, A. A., and C.-H. Moeng, 1991: Eddy diffusivity and countergradient transport in the convective atmospheric boundary layer. J. Atmos. Sci., 48, 1690-1698, https://doi.org/ 10.1175/1520-0469(1991)048<1690:EDACTI>2.0.CO;2.

Imai, K., T. Nakagawa, and H. Hashiguchi, 2007: Development of tropospheric wind profiler radar with Luneberg lens antenna (WPR LQ-7). SEI Tech. Rev., 64, 38-42, http://global-sei.com/ tr/pdf/energy/64-06.pdf.

Kaimal, J. C., J. C. Wyngaard, D. A. Haugen, O. R. Coté, and Y. Izumi, 1976: Turbulence structure in the convective boundary layer. J. Atmos. Sci., 33, 2152-2169, https://doi.org/ 10.1175/1520-0469(1976)033<2152:TSITCB > 2.0.CO;2.

Kantha, L., and H. Luce, 2018: Mixing coefficient in stably stratified flow. J. Phys. Oceanogr., 48, 2649-2665, https:// doi.org/10.1175/JPO-D-18-0139.1.

- D. A. Lawrence, H. Luce, H. Hashiguchi, T. Tsuda, R. Wilson, and M. Yabuki, 2017: Shigaraki UAV Radar Experiment (ShUREX): Overview of the campaign with some preliminary results. Prog. Earth Planet. Sci., 4, 19, https:// doi.org/10.1186/s40645-017-0133-x.

Kim, L.-S., and B. H. Kwon, 2019: Estimation of sensible heat flux and atmospheric boundary layer height using an unmanned aerial vehicle. Atmosphere, 10, 363, https://doi.org/10.3390/ atmos10070363.

Kumar, K. K., and A. R. Jain, 2006: L band wind profiler observations of convective boundary layer over Gadanki, India (13.5 $\left.{ }^{\circ} \mathrm{N}, 79.2^{\circ} \mathrm{E}\right)$. Radio Sci., 41, RS2004, https://doi.org/ $10.1029 / 2005$ rs003259.

Lenschow, D. H., 1974: Model of the height variation of the turbulence kinetic energy budget in the unstable planetary boundary layer. J. Atmos. Sci., 31, 465-474, https://doi.org/ 10.1175/1520-0469(1974)031<0465:MOTHVO > 2.0.CO;2.

_, J. C. Wyngaard, and W. T. Pennell, 1980: Mean field and second-moment budgets in a baroclinic, convective boundary layer. J. Atmos. Sci., 37, 1313-1326, https://doi.org/10.1175/ 1520-0469(1980)037<1313:MFASMB > 2.0.CO;2.

Luce, H., L. Kantha, H. Hashiguchi, and D. A. Lawrence, 2018: Turbulence kinetic energy dissipation rates estimated from concurrent UAV and MU radar measurements.
Earth Planets Space, 70, 207, https://doi.org/10.1186/s40623018-0979-1.

$-, \ldots, \ldots$, and -2019 : Estimation of turbulence parameters in the lower troposphere from ShUREX (20162017) UAV data. Atmosphere, 10, 384, https://doi.org/ 10.3390/atmos10070384.

Moeng, C.-H., and J. C. Wyngaard, 1986: An analysis of closures for pressure-scalar covariances in the convective boundary layer. J. Atmos. Sci., 43, 2499-2513, https://doi.org/10.1175/ 1520-0469(1986)043\%3C2499:AAOCFP\%3E2.0.CO;2.

, and P. P. Sullivan, 1994: A comparison of shear- and buoyancy-driven planetary boundary layer flows. J. Atmos. Sci., 51, 999-1022, https://doi.org/10.1175/1520-0469(1994) $051<0999$ :ACOSAB > 2.0.CO;2.

Peltier, L. J., and J. C. Wyngaard, 1995: Structure-function parameters in the convective boundary layer from large-eddy simulation. J. Atmos. Sci., 52, 3641-3660, https://doi.org/ 10.1175/1520-0469(1995)052<3641:SPITCB > 2.0.CO;2.

Stull, R. B., 1988: An Introduction to Boundary Layer Meteorology. Kluwer Academic, 666 pp.

Tatarskii, V. I., 1961: Wave Propagation in a Turbulent Medium. McGraw-Hill, 285 pp.

Therry, G., and P. Lacarrère, 1983: Improving the eddy kinetic energy model for planetary boundary layer description. Bound.-Layer Meteor., 25, 63-88, https://doi.org/10.1007/ BF00122098.

Tomas, S., 2007: Modélisation et étude expérimentale de la turbulence au sein des couches limites atmosphériques. Ph.D. thesis, Université Toulouse III-Paul Sabatier, 194 pp.

Troen, L., and L. Mahrt, 1986: A simple model of the atmospheric boundary layer: Sensitivity to surface evaporation. Bound.-Layer Meteor., 37, 129-148, https://doi.org/10.1007/ BF00122760.

Willis, G. E., and J. W. Deardorff, 1974: A laboratory model of the unstable planetary boundary layer. J. Atmos. Sci., 31, 1297-1307, https://doi.org/10.1175/1520-0469(1974)031,1297: ALMOTU.2.0.CO;2.

Wyngaard, J. C., and M. A. Lemone, 1980: Behavior of the refractive index structure parameter in the entraining convective boundary layer. J. Atmos. Sci., 37, 1573-1585, https://doi.org/ 10.1175/1520-0469(1980)037<1573:BOTRIS >2.0.CO;2. 